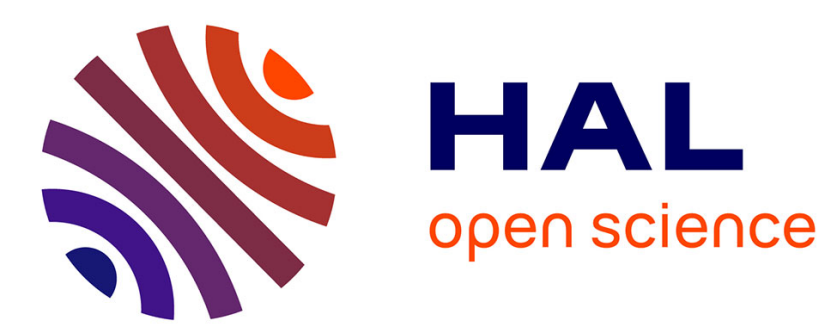

\title{
Analyse économique du droit et méthode expérimentale
}

Yannick Gabuthy, Nicolas Jacquemet

\section{- To cite this version:}

Yannick Gabuthy, Nicolas Jacquemet. Analyse économique du droit et méthode expérimentale. 2012. halshs-00746617v1

\section{HAL Id: halshs-00746617 \\ https://shs.hal.science/halshs-00746617v1}

Preprint submitted on 5 Nov 2012 (v1), last revised 11 Mar 2015 (v2)

HAL is a multi-disciplinary open access archive for the deposit and dissemination of scientific research documents, whether they are published or not. The documents may come from teaching and research institutions in France or abroad, or from public or private research centers.
L'archive ouverte pluridisciplinaire HAL, est destinée au dépôt et à la diffusion de documents scientifiques de niveau recherche, publiés ou non, émanant des établissements d'enseignement et de recherche français ou étrangers, des laboratoires publics ou privés. 


\title{
Analyse économique du droit et méthode expérimentale
}

\author{
Yannick Gabuthy ${ }^{+}$ \\ Nicolas Jacquemet
}

Pour lutter contre les activités criminelles, est-il plus efficace d'intensifier les contrôles ou d'accentuer la sévérité des sanctions? Le libre jeu du marché est-il suffisant pour allouer efficacement les droits de propriété dans une économie? La branche de l'analyse économique consacrée aux règles de droit fournit des réponses précises à ces questions. La portée de ces résultats théoriques dépend de leur pertinence empirique. Toutefois, la confrontation aux données est particulièrement délicate dans ce domaine : de nombreux comportements échappent à l'observation ou sont disponibles sous des formes qui rendent difficile toute interprétation.

La méthode expérimentale consiste à créer un environnement microéconomique contrôlé, permettant d'observer le comportement d'individus réels interagissant au sein de cet environnement. Les données empiriques collectées dans ce cadre peuvent ainsi être confrontées aux prédictions issues de l'analyse théorique. L'objectif de cet article est d'illustrer cette démarche et les principaux enseignements que l'on peut en tirer dans le domaine de l'économie du droit.

L'article débute par une présentation du déroulement d'une expérience en laboratoire, de la phase de recrutement des participants jusqu'à leur rémunération en fin de session expérimentale. L'intérêt majeur de la méthode expérimentale est qu'elle permet de s'assurer que les variations de comportement sont bien imputables aux propriétés des environnements eux-mêmes et non à des causes tierces qui échapperaient à l'observation. Cette condition est fondamentale pour assurer la fiabilité d'un lien causal observé entre le contexte juridique et les comportements des individus qui évoluent dans cet environnement.

\footnotetext{
${ }^{+}$CRESE, Université de Franche-Comté.

*École d’Économie de Paris et Université Paris I Panthéon-Sorbonne.

E-mail : yannick.gabuthy@univ-fcomte.fr.
}

Nous tenons à remercier Hélène Erkel-Rousse, l'éditrice, ainsi que deux rapporteurs anonymes pour leurs remarques précieuses sur une version antérieure de l'article. Nicolas Jacquemet tient à remercier l'Institut Universitaire de France pour son soutien financier et Ivan Ouss pour son aide dans la mise en forme du manuscrit. 
L'apport de la méthode expérimentale à la compréhension empirique de ce lien est illustré à travers quatre applications emblématiques : le théorème de Coase, le droit et la politique de la concurrence, la résolution des litiges et, enfin, les comportements criminels et leur répression.

Le théorème de Coase stipule que «dans un monde où les coûts de transaction sont nuls et les droits de propriété clairement définis, le libre jeu de la négociation aboutit à une allocation optimale des ressources, indépendante de l'attribution initiale des droits ». Ce mécanisme implique en particulier que l'intervention de l'État doit se limiter à instaurer et protéger une certaine répartition légale des droits de propriété, le libre jeu du marché permettant aux agents économiques, dans ce cadre, d'atteindre une solution efficace. Étant données ses implications en termes de politique publique, la question de la validité de l'approche coasienne apparaît primordiale.

Au-delà de cet environnement sans friction, nous nous intéressons également à l'environnement juridique qui régit les règles de concurrence. La situation de concurrence pure et parfaite, qui conduit les producteurs à sélectionner les quantités pour lesquelles le coût marginal de production et le prix de vente concordent, rend maximale la richesse produite dans l'économie. Cette propriété d'optimalité explique la préoccupation des autorités publiques à l'égard du respect de la concurrence, alors même que les producteurs sont incités à s'en éloigner puisque leurs profits sont nuls dans ce cas. L'adoption de stratégies de collusion de la part des producteurs, stratégies qui consistent à coordonner leurs comportements de manière à augmenter leurs profits, est mise en évidence dans un cadre expérimental de concurrence imparfaite. Ce cadre permet par ailleurs de mettre en exergue le rôle potentiel de la structure du marché, telle que son degré de concentration, dans l'émergence de ces accords collusifs. La concentration du marché devient ainsi un paramètre économique dont les autorités doivent tenir compte afin d'accroître leur capacité à détecter l'existence de stratégies anticoncurrentielles et d'améliorer l'efficacité de la politique de la concurrence.

La troisième thématique porte sur l'analyse économique des conflits juridiques et vise à expliquer l'occurrence des jugements ainsi qu'à analyser le comportement stratégique des justiciables lors de tels litiges. L'intérêt de ces approches est de se départir du raisonnement "coasien», selon lequel les conflits devraient être directement résolus par les parties dans la mesure où l'arrangement amiable constitue 
par nature une issue mutuellement avantageuse.

Dans une même volonté d'étudier des situations de déviation à l'égard du droit, le dernier thème vise à mettre en évidence les apports de la méthode expérimentale dans le domaine de l'analyse économique de la criminalité. L'objectif est d'analyser les déterminants de la décision individuelle de se livrer à une activité criminelle et d'étudier la pertinence des politiques répressives fondées sur la détection et la sanction.

Ces quatre champs thématiques permettent un survol des réfutations, confirmations et nuances apportées aux résultats théoriques par l'analyse expérimentale. Dans une optique normative, une conséquence possible de ce processus de réfutation empirique serait de recommander des amendements ou, a contrario, de confirmer la pertinence des règles juridiques. Néanmoins, cette aptitude de la méthode à constituer un outil d'aide à la décision au service des politiques publiques dépend crucialement de la capacité des résultats produits en laboratoire à prédire les comportements observés dans un environnement réel. Cette question liée à la notion de validité externe des résultats expérimentaux ouvre la partie conclusive de l'article, partie qui permet également de discuter des différentes directions que les politiques publiques peuvent prendre au regard de ces résultats. 
L'analyse économique des relations entre individus repose sur des modèles théoriques, qui sont des descriptions épurées et formalisées de la situation que l'on cherche à comprendre. Ces modèles recourent à un certain nombre d'hypothèses, censées rendre compte de l'environnement des décisions (les institutions) ainsi que des motivations des individus qui les prennent. L'analyse économique produit ainsi des prédictions quant au comportement d'équilibre, c'est-àdire à la situation qui devrait prévaloir dans le monde décrit par le modèle. Que la théorie se fixe un but de prévision ou d'explication, la pertinence de ces analyses dépend en grande partie de leur contenu empirique : la théorie rend-elle compte de façon satisfaisante des comportements observés ? Selon les processus classiques de la recherche scientifique, une réponse affirmative à cette question permet de valider la théorie en lui donnant un crédit empirique, tandis qu'une réponse négative conduit à remettre en cause la théorie et/ou ses hypothèses.

Cette vision idéale de la démarche scientifique cache de grandes difficultés. La première d'entre elles naît de la nécessité de définir un critère permettant de considérer comme « satisfaisant » le lien entre prédictions théoriques et comportements réels. Un modèle étant par nature épuré et abstrait, personne ne s'attend à ce qu'il décrive de façon parfaite les comportements sociaux, qui dépendent d'une infinité de causes et de dimensions dont beaucoup sont au-delà du champ de l'économie. Résoudre ce problème revient donc à déterminer le niveau d'infidélité aux comportements réels qui rend acceptable et pertinente la simplification. Cette question méthodologique très importante, dont le traitement dépasse largement les objectifs de cet article, est résolue par le recours à l'économétrie. À partir de données brutes sur les décisions prises par différents individus dans divers environnements, l'économétrie permet de statuer sur le lien que ces deux dimensions entretiennent. Elle extrait des données des réponses quantitatives quant à l'influence de l'environnement sur les décisions, qui autorisent une part d'incertitude et d'erreur reflétant la complexité du phénomène étudié. Pour être appliquées, ces techniques requièrent des données, c'est-à-dire des observations sur les comportements et les institutions. Bien que triviale, cette condition est souvent très difficile à remplir. Par ailleurs, l'une des conditions fondamentales pour appliquer ces méthodes est de pouvoir imputer les variations de comportement observées dans différents environnements aux propriétés de ces environnements eux-mêmes, plutôt qu'à des causes tierces qui échappent à l'observation. Cette condition dite « d'exogénéité » est au cœur des difficultés rencontrées par l'analyse empirique du droit. Pour ne donner qu'un seul exemple, il serait tout à fait trompeur de se fonder sur le nombre de condamnations pour établir un lien causal entre l'efficacité du système pénal et le taux de délinquance. S'il est imaginable qu'un système pénal plus efficace permette d'augmenter le 
nombre de condamnations, il est aussi tout à fait possible qu'un système pénal inefficace tende à encourager les délinquants, faisant ainsi augmenter leur nombre. Dans ce dernier cas, l'accroissement mécanique des condamnations, lié directement à celui de la délinquance ellemême, indique la défaillance du système pénal, non son efficacité. Il est en général impossible de distinguer ces deux cas radicalement opposés à partir de la seule observation de données réelles.

Le recours à l'expérimentation en laboratoire permet de s'abstraire de ces deux difficultés majeures. Ces expériences consistent à créer dans le laboratoire un environnement microéconomique contrôlé (Smith, 1982). Cette méthode de recueil des données permet donc par nature de fournir des observations sur les comportements induits par différents environnements institutionnels. Concrètement, ces variations de l'environnement prennent la forme de différents choix des paramètres de l'expérience, appelés traitements expérimentaux. Le caractère expérimental de cette méthode offre en outre à l'analyste une batterie inégalée d'instruments de contrôle de cet environnement, qui garantissent l'exogénéité des changements observés. Comme tout domaine d'application de la microéconomie, l'analyse économique du droit consiste à modéliser les situations d'intérêt comme des jeux, c'est-à-dire des règles d'interaction dans un environnement donné, et à prédire les comportements d'un certain nombre de joueurs identifiés par leur position dans le jeu. La méthode expérimentale est conçue de façon à répliquer, aussi parfaitement que possible mais de façon empirique plutôt que théorique, cette représentation de la réalité. Il s'agit en effet d'observer le comportement d'individus réels dans le cadre d'un jeu dont les règles sont identiques à celles du modèle. Les comportements observés dans ce cadre peuvent ainsi être confrontés aux prédictions issues de la théorie. L'objectif de cet article est précisément de présenter un certain nombre d'illustrations issues de cette confrontation ${ }^{1}$. Notre article ne vise ainsi en aucun cas l'exhaustivité mais s'attache à analyser les protocoles expérimentaux développés par certains auteurs fondateurs du domaine, dans une optique principalement méthodologique.

À cet égard, la première partie de l'article est préalablement consacrée à une présentation de la méthode expérimentale, tandis que les parties suivantes s'attachent à mettre en évidence les apports de la méthode expérimentale à différents domaines majeurs de l'analyse économique du droit. La deuxième partie évalue la pertinence empirique du monde sans friction décrit par le théorème de Coase, dans lequel l'environnement juridique se limite à allouer les droits de propriété. Dans la troisième partie, nous nous intéresserons à l'analyse expérimentale des règles de droit qui régissent les règles de concurrence. Les deux derniers thèmes retenus étudient des

\footnotetext{
${ }^{1}$ Cet article est une version revue et augmentée de Gabuthy et Jacquemet (2009).
} 
situations de déviation à l'égard du droit ou des engagements pris. La quatrième partie s'intéresse ainsi aux dispositions juridiques de résolution des conflits ainsi qu'aux procédures de négociation, tandis que la cinquième partie est consacrée aux comportements criminels et à leur répression. Ces quatre parties seront l'occasion d'un survol des réfutations, confirmations et nuances apportées aux résultats théoriques par l'analyse expérimentale. Les leçons qu'il est possible d'en tirer dépendent de la pertinence, pour expliquer les comportements dans le monde réel, d'observations issues d'un environnement artificiel et contrôlé sur des décisions prises par des individus enfermés (de leur plein gré) dans un laboratoire d'économie. La réponse à cette question renvoie à la notion très importante de validité externe des résultats expérimentaux, qui ouvrira la partie conclusive de l'article. Si la validité externe des résultats obtenus n'est pas remise en question, les résultats expérimentaux peuvent être considérés comme un reflet pertinent des comportements qui prévaudraient dans le monde réel. L'analyse expérimentale, tout particulièrement lorsqu'elle est appliquée aux règles de droit, devient alors un outil d'aide à la décision au service des politiques publiques. Nous tenterons pour conclure de dresser un portrait des différentes directions que ces politiques peuvent prendre.

\section{Éléments méthodologiques}

Sans donner une description complète et approfondie des procédures utilisées pour conduire des expériences en économie, il est important pour comprendre la portée de leurs résultats de se représenter le déroulement d'une expérience type ${ }^{2}$. L'analyse théorique des relations entre individus repose en économie sur la définition de règles définissant un jeu. Sur la base des résultats de la théorie des jeux, il est alors possible de prédire le comportement des individus et leur réponse à des changements dans ces règles. Les expériences en laboratoire permettent de confronter ces résultats théoriques aux observations empiriques issues des décisions de participants à l'expérience. Les sujets sont recrutés en général à l'aide de sites internet publics annonçant la possibilité de gagner de l'argent en participant à une expérience en économie ${ }^{3}$. L'une des règles importantes est en effet que les participants obtiennent une rémunération, dont

\footnotetext{
${ }^{2}$ Pour une introduction plus détaillée, le lecteur peut se reporter à Eber et Willinger (2005), Chapitre 1. Notre présentation du déroulement d'une expérience en laboratoire fait référence à l'installation la plus courante : celle d'un laboratoire dédié équipé de postes informatiques reliés à un central. Il reste tout à fait possible, cet article en décrira quelques exemples, de réaliser des expériences à l'aide d'une installation plus légère. Dans ces expériences, dites de type «papier/crayon », les postes informatiques sont remplacés par des formulaires écrits sur lesquels les participants indiquent leurs réponses, qui sont récoltées et traitées manuellement par l'expérimentaliste.

${ }^{3}$ Voir, par exemple, le site de recrutement du laboratoire d'économie expérimentale de l'Université Paris 1 Panthéon-Sorbonne (http://leep.univ-paris1.fr/accueil.htm).
} 
le montant dépend des décisions prises au cours de l'expérience. Les préférences des participants de l'expérience à l'égard des différents états du monde possibles dans le jeu sont ainsi comparables à celles que suppose le modèle théorique. Le nombre requis de participants est convoqué et se présente au laboratoire le jour de l'expérience. Le « laboratoire » est une salle équipée d'autant d'ordinateurs que de participants. Une fois installés, les participants se voient distribuer un document décrivant le déroulement de l'expérience ainsi que les instructions du jeu. Ce document donne notamment toutes les explications permettant aux joueurs de calculer leur rémunération en fonction de leurs décisions. Lorsque le jeu commence, un ordinateur central gère le déroulement de l'expérience à travers des envois d'information à chaque participant et recueille les décisions prises. Les participants prennent connaissance de ces informations et prennent leurs décisions directement sur l'écran de l'ordinateur qui leur a été attribué. À la fin de l'expérience, chaque participant reçoit, de manière privée, un paiement en liquide calculé selon les règles décrites dans les instructions.

Ces procédures offrent à l'analyste un contrôle parfait sur l'environnement des décisions et l'information des joueurs, entièrement déterminés dans la phase de conception de l'expérience. En adoptant une classification célèbre et désormais largement admise, on peut distinguer trois types d'objectifs auxquels la démarche expérimentale permet de répondre sur cette base (Roth, 1988). Elle fournit, d'abord, des tests empiriques directs de la théorie par confrontation entre les comportements prédits dans l'environnement défini par le modèle et les comportements observés dans la réplication expérimentale de ce même environnement. Il existe cependant un grand nombre de situations sociales qui restent soit ignorées soit inexpliquées par l'analyse théorique. Les expériences en laboratoire peuvent alors permettre de produire des connaissances nouvelles, en apportant des éléments empiriques quant au comportement des individus dans ces situations. À l'intersection de ces deux types d'investigation, l'économie expérimentale apparaît enfin comme un outil d'aide à la décision et à l'élaboration des institutions, permettant soit de guider la mise en pratique des résultats théoriques validés empiriquement, soit d'évaluer empiriquement l'effet sur les comportements d'institutions nouvelles ou de changements institutionnels qui échappent à l'analyse théorique.

La capacité d'une expérience à satisfaire ces objectifs dépend de façon fondamentale de sa validité interne, c'est-à-dire de la garantie que les comportements des participants sont bien le produit de l'environnement choisi - et donc observé - par l'expérimentaliste, et non d'éléments extérieurs qui échappent à l'observation. C'est en effet sous cette seule condition qu'il est 
possible d'imputer aux règles institutionnelles étudiées les changements de comportement observés. Ce souci conditionne assez largement la mise en œuvre pratique des expériences.

Une première conséquence concerne le traitement du contexte social associé à la situation à laquelle on s'intéresse. Par définition, la perception individuelle des situations de décision (relation d'emploi, transaction illégale, spéculation, etc.) et la charge morale qui leur est associée sont des dimensions qui échappent à l'observation et influencent de façon importante les décisions prises. Quelle que soit leur importance sur le plan empirique, ces questions dépassent le champ de l'analyse économique et sont donc en général laissées de côté par les études théoriques. En conséquence, la plupart des expériences sont décrites de façon décontextualisée, en utilisant un langage neutre et qui évite autant que possible de faire référence à la situation réelle que le jeu reflète. Aussi surprenant que cela puisse paraître a priori, les expériences consacrées aux comportements criminels, qui feront l'objet de la cinquième partie, sont ainsi souvent décrites en termes de simples gains et pertes, sans référence à la notion de légalité. Néanmoins, lorsque le jeu est particulièrement complexe, ces préoccupations peuvent entrer en conflit avec celle, au moins aussi importante, de la bonne compréhension des instructions. Dans certains cas (tels, par exemple, que les jeux de marché qui seront décrits dans la partie consacrée à la politique de la concurrence), la décontextualisation est ainsi abandonnée afin de simplifier la présentation du jeu.

Pour les mêmes raisons, les protocoles expérimentaux visent en général à préserver l'anonymat des participants pendant le déroulement de l'expérience. Les participants sont ainsi isolés les uns des autres (à l'aide de cloisons séparant les postes de travail) et ne disposent d'aucune information leur permettant de connaître l'identité de la personne avec laquelle ils interagissent. Dans ces conditions, les interactions entre les participants se limitent à celles qui ont été délibérément autorisées par l'expérimentaliste et les comportements sont indépendants d'éventuelles relations préexistantes (par exemple, d'amitié ou, au contraire, de rivalité) entre les participants de l'expérience.

Une règle de première importance, enfin, est de préserver la confiance des participants dans les règles qui leurs sont décrites. Au contraire, par exemple, de la psychologie, l'économie s'interdit ainsi de décrire aux participants des règles différentes de celles qui seront effectivement utilisées dans le cadre de l'expérience. Transgresser cette règle ferait en effet courir le risque qu'un participant découvre la supercherie et que se répande alors un doute sur l'authenticité des règles de l'expérience. Dans cette hypothèse, plus rien ne garantirait que les participants prennent effectivement leurs décisions dans l'environnement qui leur est décrit. 


\section{Encadré 1 : la parabole de Coase}

Soit une société hypothétique composée d'un cultivateur de blé et d'un éleveur de vaches, dont les exploitations sont contigües. En l'absence de clôture, les vaches de l'éleveur peuvent causer une nuisance au cultivateur en allant sur son terrain. Cette situation crée un dommage au cultivateur (dont l'évaluation monétaire est notée $D$ ), tandis que l'éleveur retire un bénéfice net (noté $G$ ) de cette situation. Si le dommage causé par les vaches de l'éleveur est supérieur au bénéfice que ce dernier en retire, il y a dommage social net $(D-G>0)$ et externalité négative au détriment du cultivateur. On suppose en outre que le coût de la pose d'une clôture est faible par rapport à l'ampleur du dommage. Coase examine deux cas impliquant chacun une certaine répartition initiale de droits de propriété :

1. les droits du cultivateur sur sa terre sont exclusifs avec obligation pour l'éleveur d'indemniser son voisin du dommage subi. Si l'éleveur est rationnel, il préférera poser une clôture à ses frais, dont le coût est faible ou négligeable (par hypothèse). L'utilité des deux parties est alors améliorée ;

2. l'éleveur dispose d'un droit de vaine pâture sur le terrain de son voisin. Sous cette répartition alternative des droits de propriété, il existe encore une solution purement privée permettant de parvenir à un optimum de Pareto puisque le cultivateur est incité à entrer en négociation avec l'éleveur en vue de lui racheter le droit de pâture. Sous les hypothèses formulées par Coase, la négociation peut aboutir à une solution améliorant la satisfaction des deux parties : le cultivateur rachète à son voisin ce droit d'usage à un prix supérieur à $G$, mais inférieur à $D$, ce qui est toujours possible dans la mesure où $D>G$.

Dans ces deux configurations, le libre jeu de la négociation permet d'atteindre l'optimum social par le système de prix, l'État n'intervenant que pour définir et attribuer initialement les droits de propriété.

\section{Théorème de Coase : l'accord parfait}

Sur la base d'éléments tirés de la jurisprudence anglaise et américaine, Coase (1960) montre que, " dans un monde où les coûts de transaction sont nuls et les droits de propriété clairement définis, le libre jeu de la négociation aboutit à une allocation optimale des ressources, indépendante de l'attribution initiale des droits » (l'encadré 1 décrit le fonctionnement d'une telle économie). Dans ce cadre, Coase rejette toute forme d'interventionnisme étatique au-delà de la seule nécessité d'imposer aux agents économiques le respect d'une certaine répartition légale des droits de propriété. Étant données les implications potentiellement fondamentales de cette assertion dans les domaines politique, juridique et économique (notamment en matière de régulation des externalités), la question de sa validité empirique est importante et a fait l'objet d'investigations expérimentales. 
Tableau 1 - Matrice de paiements des traitements à deux joueurs

Période 1

Période 2

\begin{tabular}{c|c|c||c|c|c}
\hline \hline $\begin{array}{c}\text { Chiffre } \\
\text { choisi }\end{array}$ & $\begin{array}{c}\text { Gain du } \\
\text { joueur } A\end{array}$ & $\begin{array}{c}\text { Gain du } \\
\text { joueur } B\end{array}$ & $\begin{array}{c}\text { Chiffre } \\
\text { choisi }\end{array}$ & $\begin{array}{c}\text { Gain du } \\
\text { joueur } A\end{array}$ & $\begin{array}{c}\text { Gain du } \\
\text { joueur } B\end{array}$ \\
\hline \hline 0 & 0,00 & 12,00 & 0 & 0,00 & 11,00 \\
1 & 4,00 & 10,00 & 10 & 1,00 & 10,00 \\
2 & 6,00 & 6,00 & 20 & 2,00 & 8,00 \\
3 & 8,00 & 4,00 & 30 & 4,00 & 6,00 \\
4 & 9,00 & 2,00 & 40 & 5,50 & 5,50 \\
5 & 10,00 & 1,00 & 50 & 9,00 & 4,00 \\
6 & 11,00 & 0,00 & 60 & 10,50 & 1,00 \\
& & 70 & 9,00 & 0,00 \\
\hline \hline
\end{tabular}

Source : Hoffman et Spitzer (1982), partie supérieure du tableau 1 de cet article (traitement à deux joueurs).

\section{Tests expérimentaux du théorème de Coase}

Hoffman et Spitzer (1982) proposent une évaluation expérimentale du théorème de Coase afin d'évaluer notamment les conditions sous lesquelles la négociation permet effectivement d'atteindre une solution Pareto-optimale.

Avant le début de l'expérience, chaque sujet se voit attribuer aléatoirement et définitivement le statut de joueur $A$ ou de joueur $B$. Après formation d'un groupe de joueurs $A$ et $B$, ce dernier est placé dans une salle et l'interaction se déroule en face-à-face, l'expérimentateur étant la seule tierce personne présente. Au cours de la première période d'interaction, l'un des deux joueurs ( $A$ ou $B$ ) est d'abord désigné de manière aléatoire (par le jet d'une pièce) comme étant le « contrôleur ». Le groupe doit ensuite choisir un chiffre entre 0 et 6 , chaque chiffre étant associé à un gain monétaire pour chacun des joueurs (cf. tableau 1, dont les éléments sont de connaissance commune entre les joueurs). Le contrôleur peut, s'il le souhaite, imposer le chiffre de son choix à l'autre joueur. Dans ce cas, chaque joueur obtient le gain correspondant et le jeu passe à la seconde période. Sinon, les protagonistes ont la possibilité de négocier. Le joueur non désigné comme contrôleur a la possibilité d'influencer ce dernier, en lui proposant notamment le transfert ex post d'une partie de ses gains. Dans ce cas, il est demandé aux parties de signer un accord écrit (contrat) mentionnant le chiffre choisi et le montant transféré de l'un à l'autre. Chaque joueur obtient alors le gain correspondant, qui dépend du chiffre et du transfert communément décidés. À l'issue de cette première période, la composition des groupes est modifiée de façon aléatoire (protocole strangers), de manière à éviter l'émergence d'effets de 
Tableau 2 - Matrice de paiements des traitements à trois joueurs

Période 1

Période 2

\begin{tabular}{c|c|c|c||c|c|c|c}
\hline \hline $\begin{array}{c}\text { Chiffre } \\
\text { choisi }\end{array}$ & $\begin{array}{c}\text { Gain du } \\
\text { joueur } A\end{array}$ & $\begin{array}{c}\text { Gain du } \\
\text { joueur } B\end{array}$ & $\begin{array}{c}\text { Gain du } \\
\text { joueur } C\end{array}$ & $\begin{array}{c}\text { Chiffre } \\
\text { choisi }\end{array}$ & $\begin{array}{c}\text { Gain du } \\
\text { joueur } A\end{array}$ & $\begin{array}{c}\text { Gain du } \\
\text { joueur } B\end{array}$ & $\begin{array}{c}\text { Gain du } \\
\text { joueur } C\end{array}$ \\
\hline \hline 1 & 1,00 & 7,00 & 7,00 & 1 & 0,00 & 8,50 & 8,50 \\
2 & 5,00 & 5,50 & 5,50 & 2 & 3,00 & 7,00 & 7,00 \\
3 & 10,00 & 4,00 & 4,00 & 3 & 5,50 & 5,50 & 5,50 \\
4 & 12,00 & 0,00 & 0,00 & 4 & 11,00 & 4,00 & 4,00 \\
& & & 5 & 13,00 & 0,00 & 0,00 \\
\hline \hline
\end{tabular}

Source : Hoffman et Spitzer (1982), partie inférieure du tableau 1 de cet article (traitement à trois joueurs).

réputation. La seconde période se déroule alors de manière identique à la première, seuls les choix disponibles et les gains correspondants étant modifiés.

Dans l'optique du théorème de Coase, quelle que soit la répartition initiale des droits de propriété (i.e. quel que soit le joueur auquel est attribué le statut de contrôleur), le chiffre 1 devrait être choisi dans la mesure où il maximise le gain agrégé. Par ailleurs, suivant le concept de rationalité individuelle sous-jacent à l'atteinte d'une solution coopérative, le contrôleur ne doit pas obtenir, à l'issue de la négociation, un gain inférieur à celui qu'il aurait obtenu en imposant son choix de manière dictatoriale (i.e. en refusant de négocier et en choisissant le chiffre maximisant son gain individuel). Ainsi, à titre illustratif, si nous supposons que le joueur $B$ a le statut de contrôleur, ce dernier doit obtenir un gain de 13 euros à l'issue de la négociation, tandis que le joueur $A$ obtiendra 1 euro (cet accord impliquant un transfert de 3 euros de l'individu $A$ vers l'individu $B$ suite au choix du chiffre 1 par ce dernier). Ce résultat correspond à la solution de Nash du jeu de négociation expérimental.

Il s'avère que les comportements observés dans cette expérience confirment largement cette prédiction. Les parties parviennent à atteindre une solution efficiente par la négociation et ce, indépendamment de la répartition initiale des droits de propriété : $90 \%$ des décisions prises sont Pareto-optimales au sens où elles maximisent le gain agrégé.

Le théorème de Coase repose néanmoins sur certaines hypothèses restrictives qui imposent notamment l'absence de coûts d'acquisition d'information et l'existence de coûts de transaction négligeables (rendant ainsi a priori son application impossible au cas d'externalités diffuses impliquant un nombre important de protagonistes). Le protocole expérimental de base a ainsi été modifié et différentes situations d'interaction ont été envisagées de manière à tester la sensibilité des résultats à ces hypothèses strictes : 
1) traitement partners : la composition du groupe reste identique durant toute l'expérience ;

2) information incomplète : le joueur $i(i=A, B)$ observe uniquement ses propres gains. Autrement dit, à la première période, le joueur $A(B)$ observe uniquement les colonnes 1 et 2 (1 et 3) du tableau 1 ci-dessus. Lors de la négociation, chaque joueur a néanmoins la liberté de révéler son vecteur de gains possibles à son partenaire ;

3) groupes de trois joueurs : l'interaction au sein des groupes de trois joueurs (sujets $A, B$ et C) varie selon les modalités de désignation du contrôleur :

a) contrôle unique : dans certains traitements, un seul contrôleur est désigné (de manière aléatoire). Ce dernier peut, s'il le souhaite, imposer le chiffre de son choix aux deux autres joueurs. Dans ce cas, chacun des trois individus obtient le gain correspondant (cf. tableau 2). Sinon, les joueurs non désignés ont la possibilité d'influencer le contrôleur, en lui proposant un transfert d'une partie de leurs gains, de manière à ce qu'il prenne une décision socialement préférable. Dans ce cas, un accord écrit est signé entre les trois parties, conformément à ce qui a été décrit plus haut ;

b) contrôle joint : dans certains traitements, deux contrôleurs sont désignés (de manière aléatoire). Ces contrôleurs doivent parvenir à se mettre d'accord sur le choix d'un chiffre. S'ils n'y parviennent pas, c'est le chiffre le plus faible des deux qui s'impose. Cependant, le joueur non contrôleur a la possibilité d'influencer l'équipe de contrôle de manière à ce qu'elle prenne une décision socialement préférable. Il peut notamment décider de transférer une partie des gains à l'un des deux (ou aux deux) contrôleurs. Dans ce cas, un accord écrit est également signé.

De manière générale, les résultats initiaux apparaissent robustes à ces modifications de l'environnement d'interaction: la solution maximisant le gain agrégé est très majoritairement sélectionnée par les individus dans ces différents traitements, les seules déviations observées ayant lieu dans la configuration « information incomplète - groupes de trois joueurs - contrôle joint $»^{4}$. S'agissant du partage de ce gain agrégé lors de la négociation éventuelle et des transferts consécutifs, il apparaît que les contrôleurs (détenteurs des droits de propriété) peuvent être scindés en deux groupes aux comportements distincts :

\footnotetext{
${ }^{4}$ Hoffman et Spitzer (1986) étendent l'analyse expérimentale présentée ici au cas où un nombre important d'individus sont amenés à interagir (i.e. le protocole induit une négociation possible entre 20 sujets). Les résultats de Hoffman et Spitzer (1982) restent valables: il n'y pas d'impact significatif de la taille du groupe sur les comportements individuels.
} 
i) certains sujets refusent de recevoir un montant inférieur à ce qu'ils auraient obtenu en maximisant leurs gains individuels, c'est-à-dire en imposant leurs choix de manière dictatoriale. Ce comportement est aisé à expliquer dans la mesure où il est conforme à l'hypothèse de rationalité individuelle, qui implique que les joueurs doivent parvenir à un accord mutuellement avantageux ;

ii) certains sujets proposent ou acceptent un partage égalitaire du gain agrégé. Ce comportement, qui concerne environ $60 \%$ des décisions observées, est plus complexe à interpréter dans la mesure où il peut avoir intuitivement deux motivations : il peut refléter une forme d'altruisme (ou d'aversion à l'inégalité) et/ou une crainte de la part du contrôleur de se voir sanctionné à la période suivante s'il perd son leadership. La confrontation des comportements dans les traitements en partners (dans lesquels la sanction est possible puisque les sujets interagissent de manière répétée) aux comportements dans les traitements en strangers (dans lesquels la possibilité de sanction est inexistante puisque les sujets n'interagissent qu'une seule fois) permet de montrer que les deux motivations sont à l'œuvre. Les données expérimentales révèlent que $90 \%(50 \%)$ des contrôleurs choisissent un partage égalitaire dans les traitements en partners (strangers). Cette observation suggère que les contrôleurs sont guidés à la fois par l'altruisme pur et par des motivations stratégiques sur la réaction de leurs partenaires. Cependant, l'existence d'un altruisme pur dans les traitements en strangers doit être nuancée : puisque les paiements sont rendus publics à l'issue de l'expérience et que la décision du contrôleur est observable par l'expérimentateur, les sujets sont incités à se comporter de manière moins égoïste. Bolton et Zwick (1995) mettent d'ailleurs en évidence, dans un contexte différent, l'effet potentiel de ce manque d'anonymat sur les comportements. Il n'en reste pas moins que diverses analyses expérimentales, répliquant et modifiant le protocole de base des auteurs, ont tenté d'expliquer l'émergence de ce partage égalitaire, qui peut être considéré comme une anomalie au regard des prédictions de la théorie des jeux coopératifs.

Harrison et McKee (1985) proposent deux hypothèses explicatives de l'occurrence d'un tel « partage altruiste » :

$\left(H_{1}\right)$ ce comportement pourrait être la conséquence d'une phase d'apprentissage trop succincte dans l'expérience, se déroulant seulement sur deux périodes et ne permettant pas aux sujets d'acquérir une perception suffisamment pertinente des implications stratégiques de l'interaction. Ces derniers s'orienteraient ainsi vers un point focal impliquant une répartition égalitaire du surplus ; 
$\left(H_{2}\right)$ ce comportement pourrait être fortement sensible à la taille du surplus que les sujets sont amenés à se partager. En effet, un accroissement substantiel de ce dernier tendrait à limiter l'émergence du comportement altruiste, dans la mesure où cette augmentation rendrait le coût d'opportunité de l'altruisme plus élevé pour le contrôleur.

Les analyses menées par les auteurs, faisant varier le nombre de périodes d'interaction ainsi que la taille du profit joint, valident l'hypothèse $H_{2}$ et rejettent l'hypothèse $H_{1}$, ce qui nous amène à relativiser la robustesse de ce comportement altruiste, dont l'émergence semble significativement sensible à une variation du protocole expérimental. En effet, Hoffman et Spitzer (1985), dans une extension de leur propre expérience, montrent que ce comportement est également fortement lié au mode de répartition des droits de propriété : le fait que le statut de contrôleur soit attribué via un mécanisme aléatoire (i.e. le jet d'une pièce) implique que les sujets considèrent ce pouvoir comme moralement illégitime et se refusent à l'utiliser de manière à obtenir une part relativement plus élevée du gain agrégé. Autrement dit, les contrôleurs considèrent l'attribution du droit de propriété comme un élément les autorisant à obtenir une part avantageuse et inégalitaire du surplus uniquement s'ils estiment avoir «gagné » ce droit. Les auteurs obtiennent ce résultat en introduisant une étape préalable à la phase de négociation, étape durant laquelle les deux sujets formant un groupe doivent participer à un jeu dont le vainqueur obtient le statut de contrôleur dans la suite de l'interaction. Les données montrent que les sujets ayant ainsi acquis le statut de contrôleur sont alors significativement moins enclins à proposer un partage égalitaire que les contrôleurs désignés de manière aléatoire. Ce résultat traduit l'existence d'une conception particulière de la justice distributive obéissant aux préceptes de John Locke, selon lesquels l'individu considère qu'il mérite un droit de propriété uniquement s'il a fourni un effort pour l'obtenir.

De manière générale, au-delà de ces études expérimentales visant à focaliser l'analyse sur un élément particulier du comportement mis en lumière dans l'article de Hoffman et Spitzer (1982), cette expérience présente potentiellement certaines limites d'ordre méthodologique dont nous devons discuter. Ces limites peuvent naturellement avoir diverses implications quant à la validité externe des résultats obtenus concernant la pertinence du théorème de Coase.

\section{Analyses de robustesse}

Il apparaît tout d'abord qu'Hoffman et Spitzer (1982) n'introduisent pas de traitement de contrôle sans possibilité de négociation entre les parties. Le protocole expérimental construit ne permet donc pas d'isoler l'effet de la négociation, ni d'attribuer l'atteinte de la solution efficiente 
à l'existence de ce mécanisme de marché. Harrison et McKee (1985), cherchant à renforcer le pouvoir explicatif de l'expérience originelle, introduisent un traitement sans négociation possible et montrent que les comportements ne parviennent jamais à une issue Pareto-optimale dans cette configuration.

Par ailleurs, les tableaux 1 et 2 révèlent l'existence d'une certaine symétrie entre les fonctions de gains des sujets de type $A$ et de type $B$. Cette caractéristique peut faciliter les négociations entre les joueurs et ainsi accroître artificiellement la probabilité d'atteindre une solution socialement optimale. Coursey, Hoffman et Spitzer (1987) s'intéressent à cette question et répliquent l'expérience en introduisant des paiements asymétriques entre les joueurs. Le chiffre maximisant le gain agrégé reste néanmoins très majoritairement choisi par les sujets, ce qui tend à confirmer la validité du théorème de Coase dans ce contexte.

Enfin, il s'avère que les éléments caractérisant le protocole de communication interindividuelle introduit par Hoffman et Spitzer (1982) ne sont pas neutres sur les résultats de l'interaction entre les sujets. De manière générale, les individus négocient en face-à-face, disposent d'un temps de discussion illimité et peuvent, dans les traitements avec information incomplète, révéler leurs fonctions de gains à leur partenaire. Ces caractéristiques peuvent clairement accroître les chances qu'ont les individus de parvenir à un accord Pareto-optimal, via la quantité d'information véhiculée par un tel mode de communication et, ainsi, induire une surestimation de la validité du résultat de Coase. Cette intuition est confirmée par McKelvey et Page (2000). Ces auteurs montrent que, lorsque la communication entre les sujets se déroule en temps limité et via un terminal informatique permettant un meilleur contrôle des échanges, l'information incomplète induit un échec possible des négociations et l'émergence de solutions négociées sous-optimales. Ce résultat ne réfute pas l'existence du théorème de Coase, qui repose précisément sur l'hypothèse d'information complète. Toutefois, il affaiblit l'une des conclusions de l'expérience de Hoffman et Spitzer (1982) selon laquelle le théorème serait robuste à l'introduction d'un environnement d'information incomplète. Ce résultat va par ailleurs dans le sens du théorème de Myerson-Satterthwaite stipulant que les parties ne peuvent exploiter tous les gains de la négociation s'ils sont en situation d'incomplétude informationnelle (Myerson et Satterthwaite, 1983). Enfin, l'idée sous-jacente au théorème de Coase selon laquelle le résultat de la négociation est indépendant de la répartition initiale des droits de propriété ignore le fait qu'il peut exister une différence entre le montant qu'un individu est disposé à payer pour acquérir un droit donné et celui qu'il est prêt à accepter pour y renoncer. Cette divergence, appelée effet de dotation, est une manifestation particulière de l'aversion aux pertes selon 
laquelle les individus ont tendance à surpondérer les pertes par rapport aux gains, alors même qu'un bien est évalué comme une perte quand il est cédé et comme un gain quand il est acquis. De manière générale, l'aversion aux pertes induit que le prix de vente d'un bien est supérieur à son prix d'achat, ce qui élimine certains échanges mutuellement avantageux et remet en question le théorème de Coase. D'un point de vue expérimental, l'effet de dotation a été mis en lumière initialement par Kahneman, Knetsch et Thaler (1990), dont l'expérience a été répliquée à plusieurs reprises, confirmant la robustesse des résultats obtenus (Rabin, 1998). Nous ne développons pas davantage cette littérature, ainsi que les protocoles expérimentaux sous-jacents, dans la mesure où ils ne visent pas explicitement à tester le théorème de Coase mais centrent l'analyse sur les éléments psychologiques explicatifs du phénomène d'aversion aux pertes.

\section{Relations entre firmes : droit de la concurrence}

Le point de départ de l'analyse économique de la concurrence est le modèle walrassien de concurrence pure et parfaite, dans lequel les producteurs prennent leurs décisions de production et de tarification sans prendre en compte l'influence de ces dernières sur le fonctionnement du marché. La principale leçon de ce modèle est que cette situation concurrentielle, qui conduit les producteurs à choisir une quantité produite égalisant le coût marginal de production et le prix de vente, est efficace au sens où elle rend maximale la quantité de richesse produite dans l'économie. Toute déviation par rapport à cette situation constitue une perte nette de richesse, car l'augmentation des profits des producteurs est moins que compensée par la diminution du surplus des consommateurs. Ce résultat simple explique la préoccupation des autorités de réglementation à l'égard du respect de la concurrence - et l'inclination des producteurs à s'en départir.

Pour étudier la politique de la concurrence, le cadre d'analyse doit être élargi aux situations où les producteurs se comportent de façon stratégique, en prenant en compte l'influence de leurs décisions sur le fonctionnement du marché. Le cadre d'analyse le plus couramment adopté est celui du modèle de Cournot, dans lequel un nombre restreint de producteurs se livre une concurrence en quantité. Dans ce contexte, les quantités produites choisies par chacun des producteurs sont inférieures à celles de l'équilibre concurrentiel et les profits sont plus élevés. Cette version du modèle n'épuise pas, cependant, l'ensemble des stratégies permettant aux producteurs d'augmenter leurs profits au détriment de la concurrence. La répétition du jeu 


\section{Encadré 2 : collusion et stratégies de punition}

La date à laquelle un paiement monétaire est reçu n'est pas neutre sur la satisfaction qui est tirée de ce paiement. La prise en compte de la répétition du jeu nécessite ainsi de définir un facteur d'actualisation, noté, a qui mesure la dépréciation subie par une unité monétaire en conséquence de son décalage d'une période de temps. Sur cette base, le modèle de collusion considère un jeu de concurrence répété à horizon infini entre $n$ firmes. Le profit total de collusion correspond au profit de monopole, noté Пм $(p, q), p$ étant le prix de marché et $q$ la quantité produite. Le profit individuel de chacune des firmes lorsque toutes se conforment à la stratégie de collusion correspond ainsi à :

$$
\sum_{t=0}^{\infty} \frac{\Pi_{\mathrm{M}}(p, q)}{n} \delta^{t}=\frac{1}{1-\delta} \frac{\Pi_{\mathrm{M}}(p, q)}{n}
$$

Les firmes mettent en œuvre une stratégie de cliquet consistant à revenir à une situation concurrentielle dès lors qu'une déviation survient. Cette situation conduit à un niveau de profit total inférieur à celui de la collusion : Пр $(p, q)$. Si cette stratégie est effectivement mise en œuvre, une firme décidant de ne pas se conformer à la stratégie de collusion afin de s'accaparer l'ensemble du marché obtient ainsi un profit égal à :

$$
\Pi_{\mathrm{M}}(p, q)+\frac{\delta}{1-\delta} \frac{\Pi_{\mathrm{P}}(p, q)}{n}
$$

La stratégie de collusion tacite est donc une stratégie d'équilibre si :

$$
\frac{1}{1-\delta} \frac{\Pi_{\mathrm{M}}(p, q)}{n} \geq \Pi_{\mathrm{M}}(p, q)+\frac{\delta}{1-\delta} \frac{\Pi_{\mathrm{P}}(p, q)}{n}
$$

Dans le cas particulier où la stratégie de punition concorde avec l'équilibre de concurrence parfaite, cette condition devient: $\delta \geq 1-\frac{1}{n}$.

permet de faire appel à des stratégies plus élaborées, qui ouvrent la porte à des comportements de collusion.

\section{Analyse expérimentale de la collusion}

L'analyse théorique des jeux répétés est fondamentalement différente selon que l'horizon du jeu est fini ou infini. Si le nombre de répétitions est fini, le principe de résolution à rebours implique que l'équilibre de Nash parfait en sous-jeux concorde avec la répétition de l'équilibre de Nash du jeu statique. La répétition du jeu n'affecte aucunement, dans ce cas, les propriétés d'équilibre du jeu de concurrence. Le passage à un horizon infini, en revanche, modifie considérablement l'ensemble de stratégies à disposition des firmes. Dans ce cadre, les producteurs peuvent en effet mettre en œuvre une stratégie coordonnée destinée à augmenter les profits, appelée stratégie de collusion. Pour que cette stratégie soit effectivement appliquée par les producteurs, il faut qu'elle ne soit assortie d'aucune incitation unilatérale à dévier. En 
d'autres termes, il ne faut pas qu'une firme ait la possibilité d'améliorer son profit en adoptant une stratégie différente lorsque l'ensemble des concurrents se conforme à la stratégie de collusion. Faire en sorte que le bénéfice de la collusion soit supérieur au profit de déviation est précisément le rôle des stratégies de punition qui sont associées à ces accords de collusion. La stratégie de punition la plus couramment retenue est la stratégie dite de cliquet, qui consiste à revenir à jamais à une situation de profit nul dès lors que l'une des firmes concurrentes dévie. Une stratégie de collusion consistant pour les firmes à se partager équitablement le profit de monopole est alors mutuellement avantageuse (et constitue par conséquent un équilibre de Nash du jeu répété) dès lors que le profit de la coopération, c'est-à-dire la répétition infinie du partage du profit de monopole, excède le profit de déviation, composé du profit de monopole et de la répétition infinie du profit nul qui résulte des punitions infligées dans ce cas. La préférence pour le présent des protagonistes et la taille du cartel sont donc des déterminants de premier ordre de cet arbitrage entre coopération et déviation, puisqu'elles déterminent le bénéfice de la collusion.

L'une des leçons de l'analyse théorique des accords de collusion est ainsi qu'ils reposent de façon importante sur la répétition de la relation concurrentielle. D'un point de vue théorique, il est même crucial que l'horizon de cette répétition soit infini. L'analyse expérimentale se heurte ainsi à une difficulté importante : comment traduire en termes empiriquement testables la notion théorique d'horizon infini ? Il faut pour ce faire s'attacher à l'esprit plutôt qu'à la lettre des modèles en horizon infini. En suivant le théoricien des jeux Martin J. Osborne, on peut en effet considérer que les horizons temporels utilisés dans les analyse théoriques «capturent un fait stylisé important, à savoir le fait que l'existence d'un nombre fini et prédéfini de périodes d'interaction peut crucialement affecter le comportement individuel (considérez par exemple les derniers mois d'un mandat présidentiel ou encore le fait que les religions tentent de convaincre de l'existence d'une vie après la mort) »(Osborne et Rubinstein, 1994, p.136). Comprise de cette façon, la notion d'horizon infini recouvre alors l'idée que les joueurs ne tiennent pas compte de la fin du jeu dans leur prise de décision, tandis que celle-ci influe sur les décisions prises dans l'ensemble du déroulement du jeu lorsque l'horizon temporel est fini. Bien que cette conception ne fasse pas consensus parmi les théoriciens des jeux, elle est largement admise dans le cadre des tests expérimentaux des résultats d'économie industrielle. Sur cette base, trois types de règles de répétition du jeu sont utilisées pour conduire les participants à interpréter, sur le plan stratégique, le jeu expérimental comme étant une interaction à horizon infini : 
i) fin de jeu aléatoire - la seule information connue des participants est la probabilité que la période en cours soit la dernière. L'arrêt du jeu dépend du résultat d'un tirage aléatoire ;

ii) fin de jeu certaine mais inconnue des participants - les instructions mentionnent la répétition du jeu elle-même mais ne donnent aucune indication quant à la durée de cette répétition. La durée du jeu est fixée à l'avance par l'expérimentaliste ;

iii) fin de jeu certaine avec un horizon suffisamment long - le nombre de répétitions est fixé à l'avance et connu des participants.

Ces configurations semblent a priori très différentes entre elles. Un consensus relatif se dégage cependant au sein de la communauté scientifique pour les considérer comme équivalentes. Cette opinion est confortée par l'analyse expérimentale de Normann et Wallace (2012). Ces auteurs montrent que la règle de terminaison du jeu n'affecte pas de façon significative le comportement de coopération dans un jeu du dilemme du prisonnier. Ces solutions semblent donc neutres sur le plan comportemental et offrent autant de possibilités de mettre en œuvre des jeux à horizon infini au sein du laboratoire. Elles permettent ainsi de tester les résultats théoriques quant aux comportements anticoncurrentiels et aux outils de la politique de la concurrence.

\section{Politique de la concurrence}

La principale difficulté en matière de lutte contre les stratégies anticoncurrentielles est de définir des critères permettant de détecter ces comportements, qui sont par nature secrets. L'analyse de la structure du marché est l'outil le plus fréquemment utilisé dans la mesure où le bénéfice de la collusion est principalement fonction du nombre de firmes entre lesquelles le profit du monopole est partagé (en dehors de la préférence pour le présent, qui est un élément par nature inobservable). Toutes choses égales par ailleurs, l'adoption de stratégies anticoncurrentielles devrait ainsi être d'autant plus probable que la concentration du marché est forte. Autrement dit, dans une situation de concurrence à la Cournot, les quantités produites dans le cadre d'un accord collusif devraient être des fonctions strictement croissantes du nombre de firmes présentes sur le marché. L'analyse théorique de Selten (1973) démontre d'ailleurs que la présence de six firmes serait suffisante à garantir un fonctionnement concurrentiel. Huck, 
Normann et Oechssler (2004) proposent une évaluation expérimentale de cette question qui repose sur une méta-analyse des résultats expérimentaux existants ${ }^{5}$.

L'un des avantages importants de la méthode expérimentale est en effet de conduire à des résultats qui sont aisément reproductibles dans d'autres contextes, d'autres lieux, d'autres temps, etc. Différentes réplications d'un même protocole expérimental ou, plus largement, d'un même environnement de décision, conduisent ainsi à une accumulation de résultats empiriques permettant d'évaluer la robustesse des comportements observés. Pour ce faire, les méthodes de méta-analyse - très fréquentes dans les sciences expérimentales - constituent un ensemble d'outils permettant de réunir les résultats issus d'études différentes afin de tirer des leçons transversales sur les comportements induits par l'environnement auquel on s'intéresse. L'une des conditions importantes de validité de la démarche est bien évidemment l'homogénéité des expériences considérées. L'étude de Huck, Normann et Oechssler (2004) se limite ainsi aux expériences en laboratoire consacrées aux jeux de collusion répétés ${ }^{6}$. Sur la base des 19 études recensées, il apparaît que des comportements collusifs surviennent parfois en situation de duopole, mais s'avèrent très rares lorsque la structure est oligopolistique (nombre de firmes supérieur ou égal à 3 ).

Quel qu'en soit le bénéfice, les accords de collusion ne peuvent constituer des stratégies individuellement rationnelles qu'à condition de reposer sur des stratégies de punition crédibles et par conséquent capables de dissuader les déviations. À structure du marché donnée, cette condition requiert en particulier que les firmes soient en position d'identifier les comportements déviants de leurs concurrentes afin de déclencher le cas échéant ces stratégies de punition. L'accès à une information individuelle sur les stratégies des firmes intervenant sur le marché peut ainsi être considéré comme un facteur facilitant la mise en œuvre de stratégies de collusion. Le droit de la concurrence européen, par exemple, considère la publication des décisions individuelles comme une pratique anticoncurrentielle. Cette position ne fait cependant pas consensus. Ainsi, le droit de la concurrence américain considère ces pratiques comme neutres en l'absence d'autres preuves d'entraves à la concurrence, tandis que les autorités danoises

\footnotetext{
${ }^{5}$ Cette recension s'accompagne d'une analyse expérimentale originale faisant varier le nombre de firmes présentes sur le marché, dont les résultats confirment dans un cadre unifié les propriétés discutées ici.

${ }^{6}$ De façon plus précise, seuls sont inclus les travaux dont le protocole s'appuie sur : (i) concurrence à la Cournot ; (ii) répétition en partners ; (iii) absence de communication; (iv) information sur les quantités agrégées uniquement ; (v) information complète sur les gains; (vi) gains constants au cours de la répétition (absence d'escompte); (vii) concurrents symétriques; (viii) instructions contextualisées. Quelques différences subsistent cependant, concernant les fonctions de coût ou de gain, l'information des participants sur les gains (selon qu'est utilisé(e) une matrice ou un module de calcul), l'espace des stratégies (entre 2 et 85), le nombre de répétitions (entre 9 et 100) et la règle de terminaison du jeu.
} 
s'engagent à publier régulièrement ces informations afin de promouvoir la concurrence. La diversité de ces positions tient à des conceptions différentes du fonctionnement des marchés. Si l'on considère que les firmes recourent à des stratégies d'imitation, consistant à adapter leur comportement à celui de leurs concurrentes, l'information devient en effet une force puissante de convergence vers la situation concurrentielle. Une augmentation des quantités produites par l'une des firmes a dans ce cadre pour conséquence l'accroissement des quantités produites par l'ensemble des acteurs du marché, jusqu'à convergence vers l'équilibre concurrentiel (Offerman, Potters et Sonnemans, 2002). L'effet de l'information sur les stratégies de collusion est ainsi un problème empirique, tributaire des déterminants du comportement des firmes.

Huck, Normann et Oechssler (2000) proposent une expérience destinée à trancher entre ces hypothèses, en faisant varier le degré d'information disponible ${ }^{7}$. Seules les quantités moyennes de la période précédente sont annoncées aux firmes dans le traitement sans information, tandis que les décisions et les profits individuels sont communiqués dans le traitement qui augmente l'ensemble d'information. Les auteurs confirment d'abord l'effet de la taille du marché discuté plus haut, dans la mesure où les comportements observés sont très proches de l'équilibre de Nash du jeu. Surtout, la comparaison entre les traitements expérimentaux tend à favoriser la seconde interprétation relative à l'effet de l'information : dans les situations où le degré d'information modifie les comportements, ce changement va sans ambiguïté en direction d'un renforcement de la concurrence. Loin de servir la mise en œuvre de stratégies de collusion, la connaissance des stratégies choisies par les firmes rivales tendrait ainsi à intensifier la concurrence.

\section{Conflits juridiques : émergence et résolution}

L'analyse économique des conflits juridiques s'intéresse au comportement des justiciables lors d'un litige et s'attache notamment à expliquer l'occurrence d'un jugement alors même qu'un arrangement amiable constitue une issue mutuellement avantageuse. Suivant un raisonnement « coasien », les conflits devraient être directement résolus entre les parties dans la mesure où l'arrangement permet de dégager un surplus positif par rapport au jugement. Dans ce contexte, pourquoi des individus rationnels ne parviennent-ils pas à trouver un tel accord ex ante,

\footnotetext{
${ }^{7}$ Le protocole de l'expérience est très proche de ceux décrits ci-dessus. Les marchés sont constitués de 4 participants, jouant en partners un jeu de concurrence répété durant 40 périodes de jeu.
} 
évitant ainsi la pérennisation d'un conflit coûteux et sous-optimal ex post $?^{8}$ Cette question peut être abordée en s'intéressant à l'encadrement strictement judiciaire des transactions, les individus ayant le choix entre un règlement amiable de leur litige et un recours à la justice mais, également, en étudiant un environnement plus consensuel, dans lequel les protagonistes peuvent faire appel à des modes alternatifs de résolution des conflits.

\section{Négociation et procès}

L'analyse théorique des conflits juridiques proposée par Gould (1973) et Posner (1973) et Landes (1971) permet d'identifier les déterminants du choix entre l'arrangement amiable et le procès. Le conflit est assimilé à un accident survenant entre deux parties, la victime devenant le demandeur, tandis que l'auteur du dommage est qualifié de défendeur. Lors de la tentative de règlement amiable du litige, le demandeur (défendeur) détermine, sur la base de ses anticipations quant à l'issue du procès éventuel, le montant minimal (maximal) de sa proposition d'arrangement, la confrontation des deux propositions déterminant l'issue du litige. Dans cette optique, les auteurs expliquent l'échec des négociations et l'occurrence des jugements par l'optimisme excessif des parties quant aux paiements espérés à l'issue du jugement. Cet optimisme excessif empêche les parties de percevoir l'avantage relatif de l'arrangement par rapport au jugement, avantage principalement lié aux coûts du procès (recours aux services d'un avocat, délais d'attente concernant le jugement, etc.). Autrement dit, ce biais d'optimisme engendre une erreur de perception du surplus associé au règlement amiable du litige : l'économie de coûts issue de l'arrangement est plus que compensée par la différence de paiements que les parties espèrent obtenir lors du procès.

Dans l'optique des modèles optimistes, Priest et Klein (1984) ont développé une analyse théorique montrant que les affaires jugées ne constituent pas un échantillon représentatif de l'ensemble des conflits donnant lieu à des poursuites judiciaires. Les négociations préalables au procès constitueraient un mécanisme de filtrage, qui opérerait une sélection des litiges donnant finalement lieu à un jugement. Les auteurs se placent dans un système de responsabilité pour faute où, pour chaque affaire, il existe un standard de décision faisant référence à un niveau de précaution légal en dessous duquel l'auteur de dommages est disculpé et au dessus duquel il est déclaré coupable. Dans ce contexte, un accord amiable est possible s'il existe un intervalle de négociation positif tel que la valeur de réserve du défendeur (i.e. son offre maximale) est

\footnotetext{
${ }^{8}$ Hicks (1932) a originellement mis en évidence l'existence de ce paradoxe concernant les grèves, à savoir l'impossibilité d'expliquer l'émergence et la pérennisation de ce type de conflit sous l'hypothèse de rationalité des agents et d'information symétrique.
} 
supérieure à celle du demandeur (i.e. sa demande minimale). Les anticipations des protagonistes quant à l'issue d'un jugement jouent un rôle central dans la détermination de ces valeurs et ainsi dans l'opportunité que l'affaire considérée soit jugée. Les litiges dans lesquels les anticipations des parties ont peu de chance de converger seront amenés à être jugés, dans la mesure où cette divergence se traduit par une réduction de l'intervalle de négociation considéré. Autrement dit, les conflits pour lesquels la faute (ou l'innocence) du défendeur est difficilement identifiable (parce que son niveau de responsabilité réel est proche du niveau légal) ne seront pas résolus à l'amiable. Les parties auront ainsi vraisemblablement des opinions divergentes et le recours au juge constituera par conséquent le seul moyen de résoudre leur différend. Les affaires jugées seraient celles dont le résultat est indéterminé ex ante et pour lesquelles les parties ont a priori autant de chances de gagner le procès.

L'évaluation expérimentale effectuée par Stanley et Coursey (1990) a précisément pour objectif de tester la validité de ce résultat théorique et d'analyser le rôle des anticipations des parties sur l'issue des litiges. Avant le début de l'expérience, chaque sujet est affecté au statut de joueur $A$ (demandeur) ou de joueur $B$ (défendeur), chaque couple de joueurs $A$ et $B$ formant un groupe. À chaque période, les sujets d'un même groupe négocient sur le partage d'une dotation de 100 jetons. En cas d'accord, chaque sujet obtient la part ainsi négociée. En cas de désaccord, l'issue du litige dépend du nombre de jetons rouges, déterminé de manière exogène, présents dans une urne constituée de 100 jetons blancs et rouges. Si le nombre de jetons rouges (i.e. le niveau de responsabilité du défendeur) est supérieur à 50 (i.e. le niveau légal), le joueur $A$ reçoit les 100 jetons et le joueur $B$ ne reçoit rien (i.e. le demandeur gagne le procès). A contrario, si le nombre de jetons rouges est inférieur à 50 , le joueur $B$ obtient un gain de 100 jetons et le joueur $A$ obtient un gain nul (i.e. le défendeur gagne le procès). Dans les deux cas, les sujets doivent assumer un coût monétaire du fait de l'échec des négociations, cet élément caractérisant le coût du procès. Les parties ne sont pas informées de la distribution des jetons dans l'urne mais un certain nombre de tirages sont effectués avant négociation de manière à leur permettre de former leurs anticipations ex ante sur l'issue du litige en cas de désaccord ex post. L'expérience comporte 4 traitements qui font varier le nombre de tirages dans l'urne (i.e. le niveau d'information donnée aux sujets quant à l'issue du procès éventuel) et le coût lié à l'échec des négociations. Ce jeu expérimental est constitué de 20 périodes de décision en protocole strangers de manière à éliminer l'effet potentiel de la répétition sur la formation des anticipations. La distribution des jetons dans l'urne est modifiée à chaque période afin de simuler différents litiges. 
Les résultats expérimentaux obtenus sont relativement ambigus quant à la validité des prédictions théoriques. Tout d'abord, à coût du procès donné, l'accroissement du niveau d'information donné aux sujets entraîne une augmentation significative du taux d'accords négociés. Ceci tend à valider l'hypothèse selon laquelle les litiges dans lesquels la faute (ou l'innocence) du défendeur apparaît plus clairement sont amenés à être jugés dans une moindre proportion ${ }^{9}$. Néanmoins, par opposition aux résultats théoriques, les coûts de procès n'ont pas d'impact significatif sur le taux de jugements, à niveau d'information donné.

Malgré l'intérêt indéniable de ces analyses, les auteurs étudient les conséquences d'un optimisme postulé sans en identifier la cause. Une explication est fournie par la littérature psychologique, qui identifie l'existence d'un biais égocentrique, selon lequel l'individu a tendance à opérer une confusion entre ce qui est juste et ce qui est bénéfique pour lui ${ }^{10}$. Autrement dit, dans le contexte de la résolution des litiges, les parties chercheraient à parvenir à un accord considéré comme juste (et non à maximiser leurs gains) mais auraient des conceptions divergentes quant à la notion de justice. Dans ce cadre, les anticipations individuelles sont amenées à diverger, chacun des protagonistes estimant que le juge prendra une décision en sa faveur. Par ailleurs, lors de la phase de négociation, chaque partie considérera son point de vue comme impartial, jugera un comportement éventuellement agressif d'autrui comme moralement contestable et y répondra négativement. L'existence de ce biais, dont les conséquences néfastes sur le taux d'accords négociés ont été confirmées par plusieurs expérimentalistes (Babcock et Loewenstein, 1997), remet en cause l'hypothèse théorique de Priest et Klein (1984) selon laquelle les erreurs d'anticipations des parties seraient aléatoires, d'espérance nulle et de même écart type.

Une limite importante de ces analyses tient néanmoins aux enjeux monétaires du litige, qui sont relativement faibles et peuvent par conséquent conduire à se demander si les sujets sont suffisamment incités à traiter sans biais l'information dont ils disposent. Hoffman, McCabe et Smith (1996) répondent positivement à cette question en confirmant la robustesse du biais égocentrique à une variation des enjeux du litige. Dans ce contexte, étant donnés les effets pervers liés à l'existence de ce biais et à l'excès d'optimisme des parties, certains auteurs ont étudié l'impact que pouvait avoir l'introduction de mécanismes institutionnels visant à faciliter la convergence des anticipations individuelles. À cet égard, on peut se demander si l'introduction d'une borne supérieure dans la fixation du montant des dommages et intérêts compensatoires

\footnotetext{
${ }^{9}$ Une réplication de cette expérience effectuée par Thomas (1995) tend à confirmer ce résultat.

${ }^{10}$ À titre d'exemple, lorsqu'il est demandé à un couple marié quelle est la répartition des tâches ménagères entre les deux protagonistes, la somme de leurs estimations dépasse généralement $100 \%$ (Ross et Sicoly, 1979).
} 
pourrait avoir certaines vertus quant à l'occurrence d'accords négociés. Suivant l'analyse théorique de Priest et Klein (1984), l'existence d'un tel plafonnement doit par nature réduire l'incertitude des parties sur l'issue du procès et permettre ainsi une diminution du taux de jugements. Les expérimentations effectuées confirment ce résultat mais mettent en évidence le fait qu'il est sensible au montant du plafond (Chapman et Bornstein, 1996 ; Babcock et Pogarsky, 1999 ; Robbennolt et Studebaker, 1999). Un montant trop élevé tend au contraire à diminuer le taux d'accords amiables dans la mesure où il génère un biais d'ancrage du côté des demandeurs et avocats (qui ont tendance à fixer leurs anticipations et demandes sur ce montant) et du côté des juges (qui ajustent leurs décisions sur ce niveau maximal) ${ }^{11}$. Dans cette optique, il est possible de limiter l'impact de ce biais sur les décisions des juges en favorisant l'émergence de plusieurs points de référence différents de la seule existence du plafond. Il s'agit notamment de permettre le recours aux décisions antérieures (jurisprudence) ou encore de former des jurys constitués de plusieurs preneurs de décision (Guthrie, Rachlinski et Wistrich, 2001).

L'ensemble des analyses présentées dans cette partie est centré sur l'étude de l'alternative négociation-procès et des déterminants du choix opéré par les individus. Néanmoins, étant donné le recours croissant à des modes extrajudiciaires de résolution des litiges (tels que l'arbitrage ou la médiation), une littérature expérimentale abondante s'est intéressée à l'analyse du fonctionnement de ces mécanismes et des incitations qu'ils véhiculent.

\section{Négociation et modes alternatifs de résolution des conflits}

Les analyses présentées dans cette partie se focaliseront sur l'arbitrage, dans la mesure où la médiation n'a pas fait l'objet, à notre connaissance, d'études expérimentales. L'arbitrage est un mode extrajudiciaire de résolution des litiges qui consiste à recourir à une ou plusieurs personnes privées choisies par les parties pour obtenir une décision impérative (ayant autorité de la chose jugée). De part son intérêt, notamment en termes de rapidité et de confidentialité, ce mécanisme connaît un développement très important dans un certain nombre de domaines. Aux États-Unis, cette procédure est imposée aux fonctionnaires de police de certains États dans le cadre des négociations salariales. Elle est également utilisée en France afin de fixer l'indemnité de congédiement des journalistes professionnels dont l'ancienneté est supérieure à 15 ans. D'un point de vue scientifique, suivant l'orientation générale de la littérature relative à l'analyse

\footnotetext{
${ }^{11}$ Le biais d'ancrage traduit le fait que les individus raisonnent par rapport à un point de référence et non de manière absolue (Tversky et Kahneman, 1974).
} 
économique de la résolution des litiges, l'efficacité de l'arbitrage est étudiée selon sa capacité à générer une convergence des propositions individuelles lors de la phase de négociation préalable.

L'analyse économique s'intéresse principalement aux deux procédures les plus utilisées en France et aux États-Unis, à savoir l'arbitrage conventionnel et l'arbitrage entre offres finales. Ces procédures se distinguent fondamentalement par leurs règles de fonctionnement. Dans l'arbitrage conventionnel, la sentence arbitrale n'est sujette à aucune contrainte : l'arbitre a une totale liberté dans sa prise de décision. L'arbitrage entre offres finales, quant à lui, réduit le choix de l'arbitre, qui est contraint d'opter pour l'une des deux propositions des parties. Les analyses théoriques visent à étudier les incitations générées par ces deux mécanismes sur le comportement des parties dans la détermination de leurs propositions et de l'arbitre dans le choix de sa sentence.

Farber (1981) propose un modèle d'arbitrage conventionnel dans lequel l'arbitre est considéré comme un agent rationnel dont l'objectif est de maximiser son utilité inter-temporelle, qui dépend non seulement de sa rémunération présente mais également de sa rémunération future. Cette seconde composante repose naturellement sur son engagement éventuel pour d'autres arbitrages. Dans ce contexte, l'arbitre, cherchant à maximiser la probabilité d'être embauché dans le futur, prend des décisions qui ne le discréditent pas aux yeux de l'une ou l'autre des parties. Cette recherche du meilleur compromis conduit l'arbitre à tenir compte assez fortement des propositions individuelles. Il peut notamment être amené à adopter un comportement égalitaire consistant à « couper la poire en deux », à savoir partager la différence entre l'exigence du demandeur et l'offre du défendeur. Cette formulation de la sentence arbitrale tend à créer ce que les auteurs appellent un effet de glaciation, i.e. à inciter les parties à formuler des propositions extrêmes de manière à maximiser leurs gains à l'issue de l'arbitrage. Le demandeur exige un montant élevé et le défendeur offre une somme faible. La présence de l'arbitre, «dans l'ombre des négociations », tendrait ainsi à exacerber le conflit et favoriserait l'échec des négociations, même s'il est à noter que l'ampleur de ce phénomène dépend du comportement des parties vis-à-vis du risque. L'incertitude inhérente à la décision arbitrale tend effectivement à rendre des individus averses au risque plus enclins à la conciliation.

Suivant le mécanisme d'arbitrage entre offres finales (Farber, 1980), l'arbitre est contraint de choisir l'une des deux propositions émises par les parties et l'objectif de cette restriction est précisément d'éliminer l'effet de glaciation en incitant les protagonistes à formuler des propositions convergentes. D'un point de vue stratégique, chaque partie est face à un dilemme : une proposition peu conciliante est synonyme de gains élevés mais a une probabilité 
relativement faible d'être sélectionnée par l'arbitre, tandis qu'une offre raisonnable procure des gains inférieurs mais a davantage de chances d'être retenue. En d'autres termes, l'argument en faveur de l'arbitrage entre offres finales, comparativement à l'arbitrage conventionnel, peut être résumé ainsi (Rehmus, 1979, p. 218) : «Si l'arbitre était contraint de choisir l'une des offres finales des parties sans être en mesure de chercher un compromis entre elles, il est probable que la logique d'une telle procédure inciterait les individus à être plus conciliants de manière à remporter la sympathie de l'arbitre. De par la convergence de leurs propositions, les parties parviendraient à terme à un accord par elles-mêmes. »

Le principal objectif des études expérimentales réalisées dans ce domaine est de tester l'existence de l'effet de glaciation et, plus largement, d'analyser l'impact de la procédure d'arbitrage considérée sur le comportement de négociation des parties et l'occurrence d'un accord amiable. Ashenfelter, Currie, Farber et Spiegel (1992) étudient les comportements induits par chacune des procédures d'arbitrage, selon le protocole expérimental suivant. Avant le début de l'expérience, chaque sujet se voit attribuer le statut de joueur $A$ (demandeur) ou de joueur $B$ (défendeur), chaque couple de joueurs $A$ et $B$ formant un groupe. À chaque période, les sujets d'un même groupe négocient sur le partage d'un surplus de 500 jetons. En cas d'accord, chaque sujet obtient la part ainsi négociée. En cas de désaccord, l'issue du litige dépend du traitement considéré. Le premier traitement consiste en un traitement de contrôle dans lequel les parties n'ont pas la possibilité de recourir à l'arbitrage en cas d'échec des négociations (leurs gains sont nuls dans ce cas). Le second traitement introduit tout d'abord une phase de négociation sans possibilité d'arbitrage (répliquant le premier traitement) suivie d'une phase de négociation avec arbitrage conventionnel en cas d'impasse. Dans le dernier traitement, la phase de négociation sans possibilité d'arbitrage est suivie d'une phase de négociation avec arbitrage entre offres finales en cas de désaccord. La décision arbitrale est caractérisée par une variable aléatoire tirée dans une fonction de distribution de loi normale sur l'intervalle $[100,500]^{12}$. Cette distribution est définie de manière à ce que sa moyenne (300) soit différente de la moitié du surplus (250), afin d'éviter qu'un partage égalitaire émerge comme un point focal dans la négociation. Les parties sont informées de la loi de distribution et 100 tirages sont effectués avant négociation de manière à leur communiquer les dernières décisions arbitrales. Par ailleurs, les auteurs

\footnotetext{
${ }^{12}$ Le fait de simuler le comportement de l'arbitre comme une variable aléatoire (tirée dans une distribution de loi normale et de connaissance commune) est fondé sur le mode de désignation de l'arbitre tel qu'il se déroule en pratique. Le processus de sélection des arbitres par les parties implique que ces derniers peuvent être considérés comme «statistiquement échangeables » : même si différents arbitres sont amenés à prendre différentes décisions dans des affaires similaires, il est pertinent de considérer ces différences comme non significatives (étant donné que tout arbitre est incité à rechercher le meilleur compromis). Cette propriété est corroborée par les enquêtes de terrain (Ashenfelter et Bloom, 1984).
} 
introduisent, au sein des traitements avec arbitrage, une variation du coût monétaire de la procédure et du degré d'incertitude auquel sont confrontés les sujets quant à la décision potentielle de l'arbitre, à travers une modification de la variance de la distribution. Ce jeu expérimental est constitué de 20 périodes de décision (20 périodes de négociation dans le premier traitement, 10 périodes de négociation sans arbitrage puis 10 périodes de négociation avec arbitrage dans les deuxième et troisième traitements). La manière dont la décision arbitrale est mise en œuvre, en cas d'impasse dans la négociation, dépend naturellement du traitement considéré. Dans le traitement 2 (i.e. traitement avec arbitrage conventionnel), la décision arbitrale correspond simplement au nombre issu du tirage aléatoire, dans la mesure où l'arbitrage conventionnel suppose que l'arbitre a une totale liberté dans sa prise de décision. Dans le traitement 3 (i.e. traitement avec arbitrage entre offres finales), la décision arbitrale correspond à la proposition individuelle, émise en amont, qui se trouve être la plus proche du nombre tiré aléatoirement. L'arbitrage entre offres finales implique en effet que l'arbitre doive choisir entre les propositions faites par les parties en conflit.

Les résultats expérimentaux obtenus apparaissent relativement ambigus au regard des prédictions théoriques. D'une part, dans les deuxième et troisième traitements, les taux d'accords les plus élevés sont observés lors de la phase de négociation, à savoir durant les dix premières périodes de l'expérience. Ce résultat tend à confirmer l'existence théorique de l'effet de glaciation, selon lequel la possibilité de recourir à la procédure d'arbitrage rend le désaccord moins coûteux pour les parties et diminue leurs incitations à la conciliation. En effet, lors de la phase de négociation sans arbitrage possible, le coût lié à l'échec de la négociation est particulièrement élevé pour les parties, puisque leurs gains sont nuls dans ce cas, alors que ce coût diminue en cas de recours à l'arbitrage, puisque leurs gains deviennent alors positifs. D'autre part, contrairement aux conclusions théoriques, il apparaît que l'arbitrage entre offres finales n'induit pas un taux d'accords plus élevé que l'arbitrage conventionnel : le taux de conflits est de $38,1 \%$ dans la première procédure et de $33,8 \%$ dans la seconde. Ce dernier chiffre cache néanmoins une certaine disparité, dans la mesure où l'efficacité de l'arbitrage conventionnel dépend du degré d'incertitude auquel sont confrontées les parties quant à la décision arbitrale. Lorsque la variance de la distribution est faible, le taux de conflits est de 41,4 \% tandis qu'il est de 28,4\% dans un environnement plus incertain, ceci suggérant que les sujets expérimentaux sont majoritairement averses au risque. Cette meilleure performance relative de l'arbitrage conventionnel est confirmée par plusieurs études expérimentales récentes 
(Dickinson, 2003; Deck et Farmer, 2007) ${ }^{13}$. Cet effet entraîne également une forme de dépendance à l'arbitrage (narcotic effect) : le fait d'avoir eu recours à l'arbitrage dans le passé incite les parties à y recourir à nouveau dans le futur (Bolton et Katok, 1998).

De manière générale, l'ensemble de la littérature présentée dans cette partie tend à confirmer les conclusions de Smith (1962), selon lesquelles les règles de fonctionnement du marché déterminent le comportement des agents économiques. Le mécanisme de résolution des litiges considéré et la prise de décision en incertitude qu'il implique potentiellement constituent l'environnement dans lequel les protagonistes interagissent et orientent pleinement leurs comportements stratégiques. Du point de vue de la politique économique et législative, il apparaît ainsi fondamental d'avoir conscience de ce phénomène et des conclusions mises en évidence par les analyses théoriques et empiriques afin d'améliorer l'efficience de l'encadrement judiciaire et extrajudiciaire des transactions.

\section{Crime et Châtiment}

Le modèle de Becker (1968) identifie les déterminants de la décision rationnelle de se livrer à une activité criminelle. Les décisions individuelles résultent dans cette perspective d'une analyse coût/bénéfice de l'environnement induit par le système pénal. Tout ce qui participe à accroître le coût de l'activité criminelle est ainsi dissuasif. Les réformes du droit pénal constituent autant de variations dans l'environnement des décisions qui pourraient permettre d'évaluer empiriquement la pertinence de ce modèle. Un premier obstacle à ce type de démarche empirique est l'existence de problèmes de simultanéité. Si une activité criminelle élevée tend à provoquer une augmentation des moyens mis au service de la détection alors, sur données en coupe, la corrélation entre ces deux quantités est artificiellement positive. Dans l'éventualité où les données permettent de surmonter cette difficulté (en utilisant, par exemple, des observations décalées dans le temps), l'analyse empirique de la criminalité se heurte également à des problèmes d'observation, dans la mesure où seuls les comportements criminels détectés sont en général observés.

\footnotetext{
${ }^{13}$ Cet effet apparaît également dans d'autres contextes. Gabuthy, Jacquemet et Marchand (2008) et Gabuthy et Jacquemet (2007) mettent en évidence l'occurrence de ce phénomène dans le cadre des mécanismes électroniques de résolution des conflits dont la structure est spécifiquement adaptée au règlement des litiges issus de transactions effectuées sur internet.
} 
L'économie expérimentale est l'une des stratégies empiriques permettant de surmonter ces difficultés. Block et Gerety (1995) étudient cette question dans un jeu de collusion similaire à ceux qui ont été décrits dans la troisième partie du présent article. L'activité criminelle consiste à proposer un prix supérieur à l'équilibre concurrentiel sur un marché répété formé de trois à cinq firmes - taille de marché qui, au regard des travaux discutés dans les parties précédentes, fournit des incitations de niveau intermédiaire à la mise en œuvre de stratégies de collusion. La répétition du jeu est aléatoire, selon une probabilité connue des participants. Pour chaque participant, cinq expériences se succèdent, comprenant en moyenne trente périodes chacune. La première correspond au jeu de concurrence répété, la deuxième correspond à ce même jeu avec possibilités de communication entre les participants, tandis que les trois dernières introduisent en outre explicitement le risque de sanction encouru par l'ensemble des firmes formant un marché dès lors que le prix de marché est supérieur au coût marginal de production. Cette sanction est appliquée à l'issue d'un tirage aléatoire et prend la forme d'une réduction des gains monétaires de l'expérience. Les niveaux de l'amende imposée et de la probabilité de détection (égale à $10 \%, 25 \%$ ou $50 \%$ ) définissent 13 modalités différentes de la politique de détection, qui varie d'une période d'interaction à l'autre. Les gains monétaires de l'expérience sont calculés à partir de la somme des gains obtenus pendant l'ensemble des cinq séries de jeux. Les gains accumulés pendant les deux premières séries permettent ainsi d'imposer des sanctions élevées pendant les trois dernières, sans risque de gain négatif pour les participants.

D’un point de vue théorique, les conditions étant réunies pour qu'un équilibre de collusion émerge, l'absence de sanction pendant la première partie de l'expérience devrait conduire à l'équilibre de monopole. Si les participants se comportent selon le modèle d'espérance d'utilité, la proportion de prix de marché supérieurs au coût marginal devrait décroître avec le profit espéré imposé par la politique de détection. À profit espéré donné, la réaction des participants à une variation de la sévérité de l'amende (i.e. compensée par une variation de sens opposé de la probabilité de détection) dépend de leur degré d'aversion au risque : l'amende accroît la criminalité des participants « risquophiles » et décroît celles des participants averses au risque.

Suivant ces prédictions, les comportements illégaux observés croissent avec le profit espéré du crime, qui résulte de la combinaison entre la probabilité de détection, le niveau de l'amende et la rentabilité de l'activité criminelle elle-même. Les augmentations compensées de la sévérité de l'amende (i.e. associées à une diminution de la probabilité qui maintient constant le profit espéré) tendent à provoquer une diminution de l'activité criminelle, comportement qui suggère des préférences d'aversion au risque. Ces premiers résultats semblent encourageants quant à la 
validité du modèle de crime rationnel à la Becker. Si les criminels se comportent effectivement selon le processus de décision décrit par le modèle d'espérance d'utilité, une meilleure connaissance de la forme de ces préférences devient un élément clé de l'analyse des comportements criminels. Pour ce faire, les auteurs proposent un second traitement, où la même expérience est reproduite sur un groupe de criminels. Les conclusions précédentes restent largement valides. Une différence importante tient cependant à leur attitude à l'égard du risque. Les criminels participant à l'expérimentation répondent à une augmentation compensée de la sévérité de l'amende par un accroissement des activités criminelles, se révélant ainsi « risquophiles ». Or, la politique optimale du modèle de Becker implique de faire tendre la probabilité de détection vers zéro et l'amende vers l'infini, car l'intensité de la détection est la dimension la plus coûteuse de la politique de répression. Les résultats expérimentaux nuancent cet arbitrage optimal entre probabilité et sanction, dans la mesure où l'intensité de la détection s'avère mieux à même de dissuader les activités criminelles de la part d'individus qui ont un goût pour le risque. Afin d'utiliser au mieux les ressources consacrées à cette activité, la méthode expérimentale permet ainsi d'évaluer l'efficacité relative de différentes configurations des politiques de détection.

\section{Politiques de détection alternatives}

En raison de sa simplicité et de ses conséquences directes en matière économique, l'évasion fiscale est le champ d'application privilégié de l'analyse économique du crime. Dans ce cadre, l'activité criminelle se réduit à un choix entre deux loteries : une loterie certaine correspondant à la légalité et une loterie risquée, dont le revenu est supérieur ou non à la précédente selon que l'activité illégale est détectée ou pas ${ }^{14}$

Du point de vue des politiques publiques, les accroissements de la probabilité de détection se traduisent par une augmentation des moyens en personnel comme en infrastructure et représentent donc un coût économique important. À moyens donnés, pourtant, l'efficacité de cet instrument dépend de l'influence sur les comportements du degré d'information des criminels. Sur la base d'investigations empiriques, il est ainsi possible d'améliorer le pouvoir dissuasif des instruments de détection, à moyens constants, en altérant les modalités selon lesquelles le risque de détection est rendu public. Tirant parti des travaux en psychologie qui mettent en évidence la tendance à l'optimisme des individus, Jacquemet, Rullière et Vialle $(2007,2008)$ étudient les

\footnotetext{
${ }^{14}$ Cette représentation est, par exemple, utilisée par Lefebvre, Pestieau, Riedl et Villeval (2012) qui étudient les comportements individuels face à la fraude fiscale et à la fraude sociale à l'aide de la méthode expérimentale.
} 
variations de comportement induites par différents modes de présentation de la probabilité de détection.

L'expérience débute par une étape préliminaire permettant de mesurer l'aversion au risque des sujets, à partir du protocole développé par Holt et Laury (2005). La décision de fraude fiscale est ensuite représentée par un jeu d'investissement. Chaque participant doit répartir les 20 jetons qui lui sont attribués au début de chaque période entre deux options. L'option $A$ rapporte 15 ECU (Experimental Currency Unit, devise fictive utilisée comme unité de compte dans les expériences en laboratoire) de façon certaine, tandis que l'option $B$ est risquée, rapportant 20 ECU avec une probabilité $(1-p)$ et $15-F$ ECU $(F>0)$ avec une probabilité $p$. Les traitements expérimentaux se distinguent par les modalités utilisées pour informer les participants de la probabilité de contrôle. Un premier traitement insiste sur la dimension individuelle du risque encouru en annonçant le risque de détection sous forme de probabilité : un tirage au sort détermine le gain associé à l'option $B$. Un second traitement introduit une dimension collective en utilisant le nombre d'individus contrôlés : $n$ participants sur les 20 présents sont tirés au sort et se voient appliquer le gain faible de l'option $B$. Les valeurs de $n$ et de $p$ sont choisies de façon à ce que $n=20 . p$. Chaque période de jeu fait varier la probabilité $p$ (11 niveaux) ainsi que le coût de l'option risquée, $F$ (2 niveaux). Les 22 combinaisons uniques de ces paramètres définissent autant de périodes de jeu pour chaque traitement. Les gains monétaires tirés de l'expérience sont déterminés par le tirage aléatoire de l'une des périodes de jeu, afin de neutraliser l'effet de la richesse sur les comportements dans l'expérience. D'un point de vue théorique, la décision d'investissement correspond à un choix de loterie, qui dépend des préférences des individus à l'égard du risque. Pour tout niveau de probabilité $p_{0}$, le comportement devrait ainsi rester le même lorsque le tirage désigne $n_{0}=20 . p_{0}$ participants. Cependant, sur le plan comportemental, les individus dont la perception est sujette à un optimisme relatif (i.e. qui sous-estiment la probabilité qu'eux-mêmes plutôt que d'autres individus soient victimes d'un évènement négatif) devraient percevoir un risque plus faible dans le second traitement.

Les résultats obtenus confirment ceux de Block et Gerety (1995) quant à l'effet des instruments de la politique de détection : les variations dans le profit espéré de la fraude ont l'effet attendu sur son niveau. Les comportements sont en outre très différents - à risque « objectif» donné - selon la politique de communication utilisée : l'insistance sur la dimension collective du risque tend à accroître le niveau de la fraude. Sur la base de ces résultats, 
l'efficacité du système répressif est d'autant plus forte que l'intensité des contrôles est présentée comme un risque individuel, indépendant du sort des autres individus concernés.

Outre la forme de la communication, la quantité d'information rendue publique est ellemême un levier susceptible d'affecter les comportements. Alm, Jackson et McKee (1992) étudient par exemple le rôle de l'incertitude quant aux instruments de la politique fiscale. Au cours de chaque période de l'expérience, les participants perçoivent un revenu tiré aléatoirement selon une loi uniforme, dont ils doivent déclarer le montant à l'expérimentaliste. Ce revenu est alors taxé de façon proportionnelle et la partie dissimulée du revenu est soumise à un risque de détection, sanctionnée par un accroissement du taux de taxe. En comparaison de la situation de référence, où l'ensemble des paramètres est de connaissance commune, les auteurs introduisent différentes sources d'incertitude. Dans ces traitements expérimentaux, le niveau de l'amende, le taux de prélèvement ou la probabilité de détection prennent alternativement la forme de variables aléatoires centrées sur la valeur utilisée dans la situation de référence. Les comportements observés dans ce contexte suggèrent sans ambiguïté de maintenir autant que possible un voile d'ignorance quant aux modalités de la politique fiscale: toute forme d'incertitude tend à accroître le taux de déclaration, dans une proportion de $25 \%$ à $40 \%$ en moyenne.

\section{Coût moral de l'illégalité}

La politique de détection, telle qu'elle est mise en œuvre, instaure un coût destiné à compenser les bénéfices de la criminalité. Le caractère dissuasif de cet instrument dépend de la forme des préférences des criminels par l'intermédiaire de leur degré d'aversion au risque. Un coût psychologique peut s'y ajouter s'il existe un coût moral à se livrer à des activités illégales, c'est-à-dire des facteurs impliquant que la satisfaction tirée d'une activité criminelle est inférieure à celle que procurerait une transaction légale dont les bénéfices économiques seraient identiques. Pour tester l'effet de la notion d'illégalité, un certain nombre de travaux ont comparé les comportements criminels selon que le jeu est décrit en termes neutres ou en faisant référence explicitement à la notion d'activité illégale. Ces travaux concluent à l'absence d'effet sur le comportement (voir Alm, McClelland et Schulze (1992) dans le cas de la fraude fiscale et Abbink et Hennig-Schmidt (2006) pour une application à la corruption).

De manière plus générale, la perte de satisfaction ressentie en raison de la transgression d'une loi peut également dépendre de l'adhésion de l'individu à cette loi. Pour explorer cette dimension, Alm, Jackson et McKee (1992) proposent un ensemble de traitements supplémentaires dans l'expérience décrite ci-dessus, dans lesquels les taxes collectées financent 
un bien public. Chaque unité monétaire prélevée procure ainsi un gain de deux unités monétaires à l'ensemble des participants. Ce traitement permet d'établir un lien entre la collecte de l'impôt et les bénéfices qui en résultent pour les contribuables. Dans toutes les configurations de la politique fiscale, l'introduction d'un bien public tend à accroître le taux de déclaration. Au-delà de cet effet de niveau, la prise en compte de l'utilisation des fonds collectés renverse également l'influence de l'incertitude, qui a un effet négatif sur le taux de déclaration quelle que soit la variable de politique fiscale concernée. Compte tenu de ce résultat, l'incertitude apparaît ainsi comme un instrument hautement risqué, dont la capacité à améliorer l'efficacité du système de répression dépend de la perception des contribuables quant à l'usage des fonds collectés. Dans cette expérience, la présence d'un bien public crée une relation entre les participants de l'expérience, qui permet à chacun de situer sa propre contribution par rapport à celle des autres sur la base des bénéfices tirés du bien public. Il se peut donc que l'effet observé soit en partie imputable à l'influence des normes sociales, forgées par les comportements moyens adoptés par le groupe de référence des individus. Cet effet d'interaction sociale est particulièrement difficile à tester empiriquement en raison des problèmes de simultanéité qu'il engendre. Si les individus sont influencés par les comportements moyens adoptés dans leur groupe de référence, ils appartiennent eux-mêmes à ce groupe et participent par conséquent à en déterminer la moyenne. Fortin, Lacroix et Villeval (2007) étudient cette question à partir d'un jeu expérimental de fraude fiscale dans lequel les participants sont informés du comportement des membres de leur groupe. Contrairement à l'expérience précédente, les gains individuels ne sont pas directement influencés par le comportement du groupe. Sur la base de traitements économétriques permettant d'isoler l'effet propre des normes sociales, les auteurs concluent à l'absence d'effet de mimétisme.

\section{Coût moral de la légalité...}

Les activités criminelles en groupe (telles que le travail au noir ou la corruption) présentent la particularité de devoir recourir à des mécanismes de mise en œuvre alternatifs au système juridique, qui garantissent la coopération de l'ensemble des criminels impliqués. L'économie expérimentale s'est particulièrement intéressée au rôle de la confiance entre criminels dans leur capacité à mener à bien une activité illégale. Abbink, Irlenbusch et Renner (2002) testent ce mécanisme dans le cas de la corruption ${ }^{15}$. Les participants sont réunis par groupes de deux joueurs. Le jeu de corruption, répété sur 30 périodes en protocole partners, consiste en deux étapes séquentielles de décisions. Le premier joueur propose un transfert au second, qui l'accepte

\footnotetext{
${ }^{15}$ Abbink, Irlenbusch et Renner (2000) présentent une expérience similaire, appliquée au travail au noir.
} 
ou le refuse. Si le transfert est accepté, il est prélevé sur les gains du premier joueur et le gain du second joueur augmente de trois fois son montant. Le second joueur doit ensuite choisir entre deux décisions, que le montant ait été accepté (et donc encaissé) ou non : l'une lui est favorable, l'autre procure un gain élevé au premier joueur. Cette structure d'interaction capture donc les éléments d'incitation essentiels d'une situation de corruption : le corrupteur (premier joueur) remet à l'agent corrompu (second joueur) un pot-de-vin destiné à encourager cet agent à exercer en sa faveur le pouvoir discrétionnaire qu'il détient. En raison de l'illégalité de cette transaction, ce montant est versé quelle que soit la suite donnée par l'agent corrompu. C'est la raison pour laquelle, en horizon fini, l'équilibre parfait en sous-jeux consiste naturellement pour le second joueur à accepter tout transfert positif et à prendre la décision qui lui est favorable - trahissant ainsi la demande implicite du premier joueur. Anticipant cette réaction, le premier joueur devrait à l'équilibre proposer un transfert nul.

Malgré ces prédictions théoriques, qui excluent toute possibilité de corruption, les auteurs observent un nombre significatif d'accords de corruption, par lesquels le versement d'un pot-devin par le participant jouant le rôle de corrupteur est récompensé par une décision favorable de la part de l'agent corrompu. Si ces résultats confirment l'existence de mécanismes alternatifs permettant de mettre en œuvre les activités criminelles en groupe, le protocole de l'expérience ne permet pas de distinguer l'effet de la confiance de celui de la réputation induite par la répétition du jeu. Pour pallier cette lacune, Abbink (2004) propose une seconde version de cette même expérience, au cours de laquelle les joueurs ne se rencontrent qu'une fois et une seule (protocole strangers). Ce changement dans le protocole diminue sensiblement la proportion d'accords de corruption conclus dans l'expérience mais ne suffit pas à l'éliminer. La répétition de la relation criminelle est donc un facteur favorisant sa viabilité mais ne constitue pas une condition nécessaire de l'émergence de ce type de comportement ${ }^{16}$.

\section{Discussion}

À l'issue de ces applications, l'économie expérimentale apparaît comme un outil puissant permettant de surmonter les difficultés d'observation propres aux situations réelles, afin de confronter aux faits les analyses théoriques issues de l'économie du droit. Que ces observations confirment ou infirment les résultats théoriques, elles produisent un ensemble de faits stylisés sur

\footnotetext{
${ }^{16}$ Plus largement, voir Jacquemet (2006) pour une présentation plus détaillée de la littérature sur l'analyse économique de la corruption.
} 
les comportements induits par différents contextes institutionnels. Les leçons qu'il convient d'en tirer dépendent de la capacité de ces observations, issues d'un environnement abstrait et artificiel, à constituer un reflet pertinent des comportements qui prévaudraient dans un contexte non contrôlé. La réponse à cette question renvoie à la notion de validité externe des résultats expérimentaux. Si celle-ci n'est pas remise en cause, l'économie expérimentale met en évidence des déterminants du comportement qui sont à l'œuvre dans le monde réel et dans la réaction des individus aux règles de droit qui régissent leur environnement. La prise en compte de ces aspects comportementaux conduit à reconsidérer les éléments de réflexion qui fondent le développement des règles juridiques ainsi que les principes politiques sous-jacents.

\section{Validité externe des expériences}

Dans une optique juridique, le débouché naturel du processus de réfutation empirique permis par l'analyse expérimentale en laboratoire serait de recommander des amendements (ou, au contraire, de confirmer l'efficacité) des règles de droit en vigueur conformément aux effets observés. Cette conclusion n'est cependant légitime qu'à condition que les comportements qui apparaissent dans le laboratoire constituent un reflet fidèle de ceux qui émergent des situations réelles. Il s'agit ainsi de déterminer si les résultats produits dans un environnement délibérément abstrait et artificiel, permettant de garantir la validité interne, sont à même de prédire correctement les choix qui seront observés dans un environnement réel. Cette question, qui renvoie à la notion de validité externe des résultats expérimentaux, revêt une importance variable selon les objectifs assignés à l'expérience. Les travaux destinés à tester la théorie ont en effet pour souci premier de répliquer aussi rigoureusement que possible les hypothèses du modèle. La validité interne de l'expérience est alors suffisante à garantir que les résultats constituent un test empirique des prédictions de la théorie. Dans ce cas, la validité externe de l'expérience est rigoureusement identique à celle du modèle (Plott, 1982) et ne constitue pas un problème spécifique de la méthode expérimentale. Elle le devient, en revanche, de façon de plus en plus fondamentale à mesure que la méthode expérimentale est utilisée à des fins d'évaluation des politiques publiques en général, et des règles de droit en particulier (Posner, 1998). Avant d'en tirer des conséquences opérationnelles, les résultats expérimentaux doivent alors être soumis à un travail d'analyse de robustesse afin d'évaluer leur validité dans des contextes qui s'écartent de l'environnement initial. En ce sens, la validité externe est une question essentiellement empirique, qui renvoie à la pérennité des résultats observés lorsque des dimensions supplémentaires de l'environnement sont prises en compte. 


\section{Encadré 3 : validité externe des expériences, quelques résultats... expérimentaux}

Parmi les nombreuses dimensions qui distinguent l'environnement dans lequel se prennent les décisions dans le laboratoire en comparaison des situations réelles, certaines sont fréquemment invoquées pour remettre en cause la validité externe des résultats expérimentaux. Falk, Fehr et Fischbacher (2003) proposent une revue de la littérature expérimentale récente évaluant la robustesse des résultats à certains de ces changements dans le protocole. Un grand nombre de travaux, par exemple, cherchent à élargir les catégories de population qui participent aux expériences (afin de mesurer les effets d'un éventuel biais de sélection). Ces travaux font appel à des professionnels du secteur économique étudié ou élargissent tout simplement le recrutement à des catégories différentes de celle -- largement majoritaire dans les travaux expérimentaux -- des étudiants. D'une façon générale, ces travaux montrent que l'apprentissage, à savoir la connaissance et la familiarité des participants à l'égard de l'environnement auquel ils sont confrontés, influence beaucoup plus les comportements que les catégories sociales d'appartenance. Quelle que soit la population des participants, la validité externe est ainsi d'autant plus forte que les conclusions de l'expérience sont tirées de périodes de jeu tardives, qui suivent un nombre de répétitions important.

Par ailleurs, le recours largement répandu à des étudiants conduit souvent à se restreindre à des enjeux monétaires limités, allant de 10 à 15 euros pour une participation d'une heure. Les travaux qui étudient l'influence d'enjeux plus importants concluent en général à un effet sensible (à la baisse) sur la variance mais limité sur la moyenne des décisions.

Dans cette perspective, l'évaluation de la validité des résultats expérimentaux présente au premier abord un important dilemme méthodologique (Siakantaris, 2000). Les méthodes d'analyse empirique disponibles sont principalement au nombre de deux : l'économétrie sur données réelles et la méthode expérimentale. Or ce sont précisément les limites du recours aux données réelles (discutées en introduction) que la méthode expérimentale a pour objectif de contourner $^{17}$. Les interrogations quant à la validité externe conduisent ainsi à un cercle infini de remises en cause de la validité externe des expériences destinées à tester la validité de résultats originels. Afin de surmonter ces difficultés, un consensus s'est plus prosaïquement dégagé pour considérer la question de la validité externe comme un processus incrémental, au cours duquel les facteurs susceptibles de remettre en cause la validité externe des résultats sont introduits dans l'expérience jusqu'à épuisement des objections potentielles (Guala, 2002; Falk, Fehr et Fischbacher, 2003). Sur cette base, l'enrichissement progressif du protocole de l'expérience, afin de prendre en compte les dimensions les plus susceptibles d'affecter les résultats, permet d'aboutir à un ensemble solide de connaissances factuelles quant aux comportements induits par

\footnotetext{
${ }^{17}$ Notons néanmoins que, d'un point de vue méthodologique, ces deux outils sont partiellement complémentaires dans la mesure où l'économétrie est fréquemment utilisée pour traiter des données expérimentales. À cet égard, voir Montmarquette (2008).
} 
différents environnements institutionnels (l'encadré 3 présente un exemple de ce processus itératif d'enrichissement des expériences en laboratoire destiné à en évaluer la robustesse).

Cette question a été profondément renouvelée dans les années récentes, sous l'impulsion du développement des expériences de terrain, qui permettent de relâcher la tension inhérente entre recherche de validité interne et satisfaction de la validité externe (Harrison et List, 2004 ; Roe et Just, 2009). Cette méthode consiste à appliquer les principes expérimentaux à l'extérieur du laboratoire, dans la situation réelle que l'on souhaite étudier et en utilisant ses véritables acteurs. En comparaison des expériences en laboratoire, le fait de produire les résultats dans un environnement moins abstrait et moins artificiel permet de garantir une meilleure adéquation des comportements expérimentaux avec les choix qui seraient observés dans le monde réel. Par ailleurs, le recours aux expériences de terrain permet d'assurer un contrôle plus fiable de l'environnement décisionnel des participants, ce qui tend à diminuer le risque que les comportements observés soient le fruit d'éléments non contrôlés et/ou qui échappent à l'observation $^{18}$. Le coût de ce gain en validité externe, par comparaison aux expériences en laboratoire, est la perte de validité interne qui va de pair avec un contrôle moindre sur l'environnement des décisions. Cet arbitrage rend ces méthodes expérimentales fondamentalement complémentaires dans la production de faits empiriques à la fois fiables et pertinents.

\section{Biais comportementaux et politiques publiques}

De manière générale, la prise en compte des connaissances issues de l'analyse expérimentale dans l'édiction des règles de droit encadrant les comportements dépend des objectifs visés par le législateur. Les principes suivis en la matière sont traditionnellement classés en deux familles, qui s'opposent par la légitimité accordée au contrôle des comportements individuels. Les principes de type «paternaliste » tendent ainsi à favoriser les règles qui permettent d'imposer aux individus les choix considérés comme les meilleurs pour eux-mêmes par le législateur. Les principes de nature libérale, au contraire, privilégient les règles qui laissent aux individus la libre disposition de leurs choix. Chacun de ces principes repose sur des exigences très fortes. La réussite de politiques paternalistes nécessite l'existence d'un législateur à la fois omniscient disposant d'une connaissance parfaite des préférences des individus - et d'une irréprochable bienveillance à l'égard de chacun. Le libéralisme exige quant à lui que tous les individus se

\footnotetext{
${ }^{18}$ Olken (2007) propose par exemple une expérience de terrain consacrée à la question de la corruption ; voir Levitt et List (2009) pour une synthèse des travaux mobilisant cette méthode.
} 
montrent en toutes circonstances capables de prendre des décisions parfaitement rationnelles et conformes à leurs intérêts.

La prise en compte des déterminants alternatifs des comportements, autres que la seule rationalité économique, a profondément renouvelé ce débat à travers l'émergence de la notion de paternalisme libéral (Thaler et Sunstein, 2008). Tenant compte des biais de comportement observés dans le laboratoire, cette notion prend acte du principe libéral selon lequel seuls les individus sont à même d'identifier les choix les meilleurs pour eux-mêmes tout en tirant les conséquences paternalistes de ce que les individus s'avèrent en de nombreuses circonstances incapables de les adopter librement (Sunstein et Thaler, 2003). Le paternalisme libéral recommande ainsi de privilégier les règles de droit qui conduisent les individus à prendre les décisions qui seraient librement sélectionnées par eux en l'absence de ces biais (Jolls et Sunstein, 2006). Ce faisant, le paternalisme libéral amende les principes libéraux pour tenir compte des éléments psychologiques du comportement qui rendent caduque l'hypothèse libérale selon laquelle la liberté de choix conduit chacun à la situation la meilleure. Simultanément, ce principe échappe à la coercition inhérente aux politiques d'essence purement paternalistes. Il s'agit en effet non d'imposer un choix, mais d'exploiter les biais de comportement afin d'orienter les choix dans un sens considéré comme préférable d'après la perception qu'a le législateur des situations souhaitées par les individus. Qu'il soit ou non explicitement revendiqué, le paternalisme libéral imprègne nombre de décisions contemporaines de politique publique comme de dispositions législatives. Si le paternalisme recommanderait par exemple d'interdire purement et simplement la vente de tabac afin d'éviter aux individus les risques sanitaires encourus par sa consommation, le paternalisme libéral suggère quant à lui d'orienter les choix de consommation à l'aide d'un système de taxes qui, sans recourir à une interdiction formelle, est suffisamment dissuasif pour détourner les individus de pratiques qu'eux-mêmes déplorent.

Cette logique soulève pourtant de nombreux problèmes. La principale difficulté tient à la capacité du législateur à identifier ce qui, dans les choix effectifs, relève ou non de biais de comportements. Un certain nombre d'analyses, tant théoriques (Becker et Murphy, 1988) qu'empiriques (Becker, Grossman et Murphy, 1991 ; Gruber et Koszegi, 2001), suggèrent ainsi que les pratiques de nature addictive peuvent résulter d'un choix parfaitement rationnel. Dans ce cas, le paternalisme libéral impose aux choix individuels des contraintes qui vont à l'encontre de leur bien-être. Dans l'hypothèse même où les choix résultent effectivement de biais de comportements, une seconde difficulté tient à l'évaluation par le législateur de la situation souhaitable vers laquelle orienter les choix. Le paternalisme libéral se trouve donc soumis aux 
mêmes critiques qui rendent le paternalisme suspect au regard des principes libéraux (Mitchell, 2005). À l'inverse, enfin, le paternalisme libéral s'avère dans certaines situations incapable de satisfaire les objectifs assignés par des principes paternalistes : dans le cadre de dispositions de nature redistributive, le maintient d'une liberté de choix conduirait par exemple inévitablement au retrait des individus dont la situation est détériorée par ces règles (Munnell, 2003).

\section{Biais comportementaux et valeur expressive du droit}

Cet enrichissement de l'analyse des déterminants du comportement individuel face aux règles de droit s'accompagne également d'un renouvellement de la réflexion sur les éléments qui fondent le développement de ces règles et de leur respect par les individus.

Suivant l'approche traditionnelle, la décision individuelle impliquant le respect (ou l'irrespect) du droit serait le résultat d'un calcul coût/bénéfice opéré par l'agent, l'issue de ce calcul étant uniquement liée aux incitations économiques (i.e. sanction et/ou récompense) associées à la mise en œuvre de la règle. Le champ de l'analyse économique de la criminalité, développé dans la cinquième partie de l'article, en constitue une illustration. À titre d'exemple, les individus éviteraient de fumer dans les lieux publics uniquement du fait de la sanction monétaire associée au non respect de la loi Évin de 1991. L'ensemble des travaux mis en lumière dans la présente synthèse repose sur ce postulat. Or, dans une perspective plus comportementale, certaines analyses expérimentales récentes montrent que ce raisonnement est contestable dans la mesure où la règle juridique aurait une « valeur expressive » en véhiculant un contenu moral ou normatif qui fonderait son respect par les individus (au-delà des seules incitations explicites associées). Selon cette approche, le droit influencerait les décisions individuelles à travers deux canaux de transmission indépendants du mécanisme d'incitation extrinsèque lié à l'existence de la règle (Cooter, 1998).

D'une part, le droit peut constituer un mécanisme de coordination en modifiant les anticipations des individus quant au comportement des autres. Dans le cadre d'un problème de coordination où plusieurs individus doivent adopter la même action simultanément sans avoir la possibilité de communiquer au préalable, ces derniers seront a priori amenés à adopter la solution édictée par le droit, solution qui devient ainsi un point focal sur lequel les comportements se coordonneront. À titre d'exemple, la règle du code de la route imposant aux conducteurs de rouler à droite permet aux usagers de se coordonner sur cette norme, alors même que deux équilibres de Nash impliquant des gains identiques existent dans le jeu sous-jacent (i.e. 
rouler à droite ou à gauche). Cette propriété du droit a été validée expérimentalement, notamment dans le cadre du jeu de la bataille des sexes ${ }^{19}$.

Le droit peut d'autre part avoir une influence plus directe sur les préférences individuelles via les valeurs morales associées au respect de la règle juridique. L'existence de la règle juridique peut en effet «stigmatiser» un comportement comme étant inadéquat socialement et modifier ainsi les préférences individuelles vis-à-vis de son adoption. Cet aspect peut notamment être illustré par l'analyse expérimentale de Galbiati et Vertova (2008) développée dans le cadre d'un jeu de bien public. Les auteurs introduisent une règle imposant un niveau minimal de contribution individuelle, cette règle étant de connaissance commune entre les participants et associée à un mécanisme économique d'incitation (i.e. un système de contrôle avec une sanction monétaire en cas de violation de la règle ou une récompense en cas de respect de l'obligation). L'expérience fait varier le niveau d'exigence de la règle mentionnée en considérant deux niveaux de contribution minimale (i.e. niveau faible versus niveau élevé), tout en maintenant constantes les incitations associées ${ }^{20}$. Les résultats établissent notamment que la propension moyenne à contribuer augmente avec le degré d'exigence de la règle, alors même que les incitations monétaires sont identiques. Le droit (ou l'obligation) aurait ainsi une fonction expressive de par son contenu moral ou normatif, qui favoriserait l'adhésion collective. Tyran et Feld (2006) montrent par ailleurs que cette adhésion est d'autant plus forte que la règle n'est pas exogène, c'est-à-dire imposée par l'expérimentaliste, mais choisie par les agents eux-mêmes, via une procédure de referendum par exemple. Cet aspect renvoie plus généralement à la question de la légitimité du droit et de son importance comme déterminant de la capacité d'influence des règles de droit sur les comportements humains.

\footnotetext{
${ }^{19}$ Le jeu de la bataille des sexes modélise une situation d'interaction dans laquelle deux individus souhaitent exercer une activité commune mais ont des préférences divergentes quant à la nature de cette activité. Ce jeu induit à la fois une mutualité et un conflit d'intérêts entre les joueurs et comporte deux équilibres de Nash en stratégies pures caractérisant un problème de coordination sur l'activité à exercer (voir Croson, 2009, pour une synthèse des travaux portant sur l'analyse du droit comme mécanisme de coordination des comportements individuels).

${ }^{20}$ Dans le traitement « niveau faible » («niveau élevé »), le degré minimal de contribution au bien public exigée correspond à $20 \%$ de la dotation $(80 \%)$.
} 


\section{Bibliographie}

Abbink K. (2004). "Staff Rotation as an Anti-Corruption Policy: An Experimental Study", European Journal of Political Economy, vol. 43, n4, pp. 887-906.

Abbink K. et Hennig-Schmidt H. (2006). "Neutral versus Loaded Instructions in a Bribery Experiment", Experimental Economics, vol. 9, n², pp. 103-121.

Abbink K., Irlenbusch B. et Renner E. (2000). "The Moonlighting Game: An Experimental Study on Reciprocity and Retribution", Journal of Economic Behavior \& Organization, vol. 42, $\mathrm{n}^{\circ} 2,265-277$.

Abbink K., Irlenbusch B. et Renner E. (2002). "An Experimental Bribery Game", Journal of Law, Economics, \& Organization, vol. 18, n², 428-454.

Alm J., Jackson B. et McKee M. (1992).’Institutional Uncertainty and Taxpayer Compliance”, American Economic Review, vol. 82, n4, pp. 1018-1026.

Alm J., McClelland G.H. et Schulze W.D. (1992). "Why Do People Pay Taxes?", Journal of Public Economics, vol. 48, n¹, pp.21-38.

Ashenfelter O. et Bloom D. E. (1984). "Models of Arbitrator Behavior: Theory and Evidence", American Economic Review, vol. 74, pp. 111-124.

Ashenfelter O., Currie J., Farber H. S. et Spiegel M. (1992). "An Experimental Comparison of Dispute Rates in Alternative Arbitration Systems”, Econometrica, vol. 60, n6, 1407-1433.

Babcock L. et Loewenstein G. (1997). "Explaining Bargaining Impasse: The Role of SelfServing Biases," Journal of Economic Perspectives, vol. 11, n¹, 109-126.

Babcock L. et Pogarsky G. (1999). "Damage Caps and Settlement: A Behavioral Approach", Journal of Legal Studies, vol. 28, n², pp. 341-370.

Becker G. S. (1968). "Crime and Punishment: An Economic Approach", Journal of Political Economy, vol. 76, n², pp. 169-217.

Becker G.S., Grossman M. et K.M. Murphy (1991). "Rational Addiction and the Effect of Price on Consumption”, American Economic Review, vol. 81, n², pp. 237-241.

Becker G.S. et Murphy K.M. (1988). "A Theory of Rational Addiction", Journal of Political Economy, vol. 96, n 4, pp. 675-700.

Block M.K. et Gerety V.E. (1995). "Some Experimental Evidence on Differences between Student and Prisoner Reactions to Monetary Penalties and Risk", Journal of Legal Studies, vol. 24, n¹, pp. 123-138. 
Bolton G.E. et Katok E. (1998). "Reinterpreting Arbitration's Narcotic Effect: An Experimental Study of Learning in Repeated Bargaining", Games and Economic Behavior, vol. $25, n^{\circ} 1$, pp. 1-33.

Bolton G.E. et Zwick R. (1995). "Anonymity versus Punishment in Ultimatum Bargaining", Games and Economic Behavior, vol. 10, n¹, pp. 95-121.

Chapman G.B. et Bornstein B.H. (1996). "The More You Ask For, the More You Get: Anchoring in Personal Injury Verdicts", Applied Cognitive Psychology, vol. 10, n6, pp. 519540 .

Coase R.H. (1960). "The Problem of Social Cost", Journal of Law \& Economics, vol.3, pp. 144.

Cooter R. (1998). "Expressive Law and Economics", Journal of Legal Studies, vol. 27, n², pp. 585-608.

Coursey D.L., Hoffman E. et Spitzer M.L. (1987). "Fear and Loathing in the Coase Theorem: Experimental Tests Involving Physical Discomfort", Journal of Legal Studies, vol. 16, n¹, pp. 217-248.

Croson R. (2009). "Experimental Law and Economics", Annual Review of Law and Social Science, vol. 5, pp. 25-44.

Deck C.A. et Farmer A. (2007). "Bargaining over an Uncertain Value: Arbitration Mechanisms Compared”, Journal of Law, Economics, \& Organization, vol. 23, n³, pp. 547-579.

Dickinson D. (2003). "A comparison of Conventional, Final Offer, and Combined Arbitration for Dispute Resolution”, Industrial \& Labor Relations Review, vol. 57, n², pp. 288-301.

Eber N. et Willinger M. (2005). L'Économie expérimentale, Éditions La Découverte, collection Repères, Paris, 123 pages.

Falk A., Fehr E. et Fischbacher U. (2003). "On the Nature of Fair Behavior", Economic Inquiry, vol. 41, n¹, pp. 20.

Farber H.S. (1980). "An Analysis of Final-Offer Arbitration", Journal of Conflict Resolution, vol. $24, n^{\circ} 4,683-705$.

Farber H.S. (1981). "Splitting-the-Difference in Interest Arbitration", Industrial and Labor Relations Review, vol. 35, n¹, pp. 70-77.

Fortin B., Lacroix G. et Villeval M.-C. (2007). "Tax Evasion and Social Interactions", Journal of Public Economics, vol. 91, n¹1-12, pp. 2089-2112.

Gabuthy Y. et Jacquemet N (2007). "Une évaluation expérimentale des modes électroniques de résolution des litiges", Revue Économique, vol. 58, nº, pp. 1309-1330.

Gabuthy Y. et Jacquemet N. (2009). "Économie expérimentale et droit", in Analyse économique du droit, Deffains B. et Langlais E. (éds), De Boeck Universités, pp. 335-369. 
Gabuthy Y., Jacquemet N. et Marchand N. (2008). "Does Resorting to Online Dispute Resolution Promote Agreements? Experimental Evidence", European Economic Review, vol. 52, n², pp. 259-282.

Galbiati R. et Vertova P. (2008). "Obligations and Cooperative Behaviour in Public Good Games", Games and Economic Behavior, vol. 64, n¹, pp. 146-170.

Gould J.P. (1973). "The Economics of Legal Conflicts", Journal of Legal Studies, vol. 2, n², pp. 279.

Gruber J. et Koszegi B. (2001). "Is Addiction"Rational"? Theory and Evidence", Quarterly Journal of Economics, vol. 116, n 4, 1261-1303.

Guala F. (2002). "On the Scope of Experiments in Economics: Comments on Siakantaris", Cambridge Journal of Economics, vol. 26, n², pp. 261-267.

Guthrie C., Rachlinski J.J. et Wistrich A.J. (2001). "Inside the Judicial Mind", Cornell Law Review, vol. 86, n²4, pp. 1-8.

Harrison G.W. et List J.A. (2004). "Field experiments", Journal of Economic Literature, vol. 42, nº , pp. 1009-1055.

Harrison G.W. et McKee M. (1985). "Experimental Evaluation of the Coase Theorem", Journal of Law \& Economics, vol. 28, n³, 653-670.

Hicks J. (1932). The Theory of Wages, Mac Millan Press, London, 247 pages.

Hoffman E., McCabe K. et Smith V.L. (1996). "Social Distance and Other-Regarding Behavior in Dictator Games", American Economic Review, vol. 86, n³, pp. 653-660.

Hoffman E. et Spitzer M.L. (1982). "The Coase Theorem: Some Experimental Tests", Journal of Law \& Economics, vol. 25, n¹, pp. 73-98.

Hoffman E. et Spitzer M.L. (1985). "Entitlements, Rights, and Fairness: An Experimental Examination of Subjects' Concepts of Distributive Justice", Journal of Legal Studies, vol. 14, n², pp. 259-297.

Hoffman E. et Spitzer M.L. (1986). "Experimental Tests of the Coase Theorem with Large Bargaining Groups”, Journal of Legal Studies, vol. 15, n¹, pp. 149-171.

Holt C.A. et Laury S.K. (2005). "Risk Aversion and Incentive Effects: New Data without Order Effects", American Economic Review, vol. 95, n³, pp. 902-904.

Huck, S., Normann H.-T. et Oechssler J. (2000). "Does Information about Competitor's Actions Increase or Decrease Competition in Experimental Oligopoly Markets?", International Journal of Industrial Organization, vol. 18, n¹, pp. 39-57.

Huck, S., Normann H.-T. et Oechssler J. (2004). "Through Trial and Error to Collusion", International Economic Review, vol. 45, n¹, pp. 205-224. 
Jacquemet N. (2006). "Microéconomie de la corruption", Revue Française d'Économie, vol. 20, $\mathrm{n}^{\circ} 4$, pp. 118-159.

Jacquemet N., Rullière J.-L. et Vialle I. (2007). "Contrôle des activités illégales en présence d'un biais d'optimisme", Revue Économique, vol. 58, n³, pp. 555-564.

Jacquemet N., Rullière J.-L. et Vialle I. (2008). "Monitoring Optimistic Agents", Journal of Economic Psychology, vol. 29, n5, pp. 698-714.

Jolls C. et Sunstein C.R. (2006). "Debiasing through Law", Journal of Legal Studies, vol. 35, $\mathrm{n}^{\circ} 1$, pp. 199-241.

Kahneman D., Knetsch J.L. et Thaler R.H. (1990). "Experimental Tests of the Endowment Effect and the Coase Theorem", Journal of Political Economy, vol. 98, nº, pp. 1325-1348.

Landes W.M. (1971). "An Economic Analysis of the Courts", Journal of Law \& Economics, vol. $14, \mathrm{n}^{\circ} 1$, pp. 61-107.

Lefebvre M., Pestieau P., Riedl A. et Villeval M-C. (2012). "Les attitudes sont-elles différentes face à la fraude fiscale et à la fraude sociale ?", Économie et Prévision, à paraître.

Levitt S.D. et List J.A. (2009). "Field Experiments in Economics: The Past, the Present, and the Future", European Economic Review, vol.53, n 1, pp. 1-18.

McKelvey R.D. et Page T. (2000). "An Experimental Study of the Effect of Private Information in the Coase Theorem", Experimental Economics, vol. 3, n³, pp. 187-213.

Mitchell G. (2005). "Libertarian Paternalism Is an Oxymoron", Northwestern University Law Review, vol. 99, n³, pp. 152-186.

Montmarquette C. (2008). "L'économétrie des données expérimentales : défis et opportunités", Économie \& Prévision, vol.182, n¹, pp. 7-17.

Munnell A. H. (2003). "A Non-libertarian Paternalist's Reaction to "Libertarian Paternalism is Not an Oxymoron"”, Federal Reserve Bank of Boston 48th Annual Conference, 9 pages.

Myerson R. B. et Satterthwaite M.A. (1983). "Efficient Mechanisms for Bilateral Trading", Journal of Economic Theory, vol. 29, n², pp. 265-281.

Normann H. et Wallace B. (2012). "The Impact of the Termination Rule on Cooperation in a Prisoner's Dilemma Experiment”, International Journal of Game Theory, vol. 41, n³, pp. 707718.

Offerman T., Potters J. et Sonnemans J. (2002). "Imitation and Belief Learning in an Oligopoly Experiment", Review of Economic Studies, vol. 69, n 4, pp. 973-997.

Olken A. (2007). "Monitoring Corruption: Evidence from a Field Experiment in Indonesia", Journal of Political Economy, vol. 115, n², pp. 200-249. 
Osborne M.J. et Rubinstein A. (1994). A Course in Game Theory. MIT Press, Cambridge (MA), 352 pages.

Plott C.R. (1982). "Industrial Organization Theory and Experimental Economics," Journal of Economic Literature, vol. 20, n²4, pp. 1485-1527.

Posner R.A. (1973). "An Economic Approach to Legal Procedure and Judicial Administration", Journal of Legal Studies, vol. 2, n², pp. 399.

Posner R.A. (1998). "Rational Choice, Behavioral Economics, and the Law", Stanford Law Review, vol. 50, n5, pp. 1551-1575.

Priest G.L. et Klein B. (1984). "The Selection of Disputes for Litigation", Journal of Legal Studies, vol. 13, n¹, pp. 1-55.

Rabin M. (1998). "Psychology and Economics", Journal of Economic Literature, vol. 36, n¹, pp. 11-46.

Rehmus C. (1979). "Interest Arbitration", in Portrait of a Process: Collective Negotiations in Public Employment, Public employment relations services (ed.), Labor Relations Press, pp. 212268.

Robbennolt J.K. et Studebaker C.A. (1999). "Anchoring in the Courtroom: The Effects of Caps on Punitive Damages", Law and Human Behavior, vol. 23, n³, 353-373.

Roe E. et Just D.R. (2009). "Internal and External Validity in Economics Research: Tradeoffs between Experiments, Field Experiments, Natural Experiments, and Field Data", American Journal of Agricultural Economics, vol. 91, n5, pp. 1266-1271.

Ross M. et Sicoly F. (1979). "Egocentric Biases in Availability and Attribution", Journal of Personality and Social Psychology, vol. 37, n³, pp. 322-336.

Roth A.E. (1988). "Laboratory Experimentation in Economics: A Methodological Overview", Economic Journal, vol. 98, n³93, pp. 974-1031.

Selten R. (1973). "A Simple Model of Imperfect Competition, Where Four Are Few and Six Are Many", International Journal of Game Theory, vol. 2, n¹, pp. 141-201.

Siakantaris N.D. (2000). "Experimental Economics under the Microscope", Cambridge Journal of Economics, vol. 24, n³, pp. 267-281.

Smith V.L. (1962). "An Experimental Study of Competitive Market Behavior", Journal of Political Economy, vol. 70, n², pp. 111-137.

Smith V.L. (1982). "Microeconomic Systems as an Experimental Science", American Economic Review, vol. 72, n5, pp. 923-955.

Stanley L.R. et Coursey D.L. (1990). "Empirical Evidence on the Selection Hypothesis and the Decision to Litigate or Settle", Journal of Legal Studies, vol. 19, n ${ }^{\circ}$, pp. 145-172. 
Sunstein C. et Thaler R. (2003). "Libertarian Paternalism is Not an Oxymoron", University of Chicago Law Review, vol. 70, n, pp. 1159-1202.

Thaler R.H. et Sunstein C.R. (2008). Nudge: Improving Decisions About Health, Wealth, and Happiness, Yale University Press, 224 pages.

Thomas R.E. (1995). "The Trial Selection Hypothesis without the 50 Percent Rule: Some Experimental Evidence”, Journal of Legal Studies, vol. 24, n¹, pp. 209-228.

Tversky A. et Kahneman D. (1974). "Judgment under Uncertainty: Heuristics and Biases", Science, vol. 185, n4157, pp. 1124-1131.

Tyran J.-L. et Feld L. (2006). "Achieving Compliance when Legal Sanctions Are NonDeterrent", Scandinavian Journal of Economics, vol. 108, n¹, pp. 135-156. 